\title{
马铃薯资源晚疫病抗性的全基因组关联分析
}

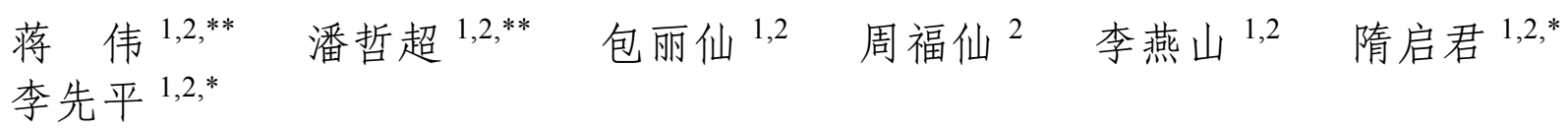

1 云南省农业科学院经济作物研究所, 云南昆明 $650205 ;^{2}$ 云贵高原马铃薯与油菜科学观测实验站, 云南昆明 650200

摘 要: 广谱抗性基因的挖掘是马铃薯高抗晚疫病品种选育的基础。本研究以 288 份国际马铃薯中心笛选的晚疫病 抗性群体为试验材料, 经过连续 2 年田间调查, 计算 AUDPC 和 SAUDPC 值, 评估群体晚疫病抗性; 利用 SLAF-seq 方法进行群体简化基因组测序, 通过对晚疫病抗性表型数据的全基因组关联分析, 挖掘晚疫病抗性相关的遗传位点 和候选基因, 为晚疫病抗性品种选育和抗病机理研究提供一定的理论和材料基础。结果表明, 晚疫病抗性在 288 份材 料间存在着广泛的遗传差异; 基于 5 种分析模型, 共鉴定到 82 个与晚疫病抗性显著关联的位点; 在关联区间关联到 54 个已知或可能与晚疫病抗性相关的基因。其中, 23 个基因为抗性基因，包括晚疫病抗性基因 $R 1$ 同源基因、 $S w-5$ 同源基因 $(R 8)$ 和 Rpi-vnt1 以及编码多效性耐药蛋白基因; 5 个基因编码 MAPK 蛋白和 WRKY 转录因子; 1 个基因参与 茉莉酸途径; 3 个基因与水杨酸途径相关; 6 个基因是病程相关的基因; 3 个基因参与苯基丙酸类合成途径; 其他与晚 疫病抗性相关的基因，如 HMGR 基因(2 个)、细胞色素 P450 (21 个)。

关键词: 马铃薯; 晚疫病抗性; 全基因组关联分析; 抗性基因; 国际马铃薯中心

\section{Genome-wide association analysis for late blight resistance of potato resources}

\author{
JIANG Wei ${ }^{1,2, * *}$, PAN Zhe-Chao ${ }^{1,2, * *}$, BAO Li-Xian ${ }^{1,2}$, ZHOU Fu-Xian ${ }^{2}$, LI Yan-Shan ${ }^{1,2}$, SUI Qi-Jun ${ }^{1,2,{ }^{*}}$, and \\ LI Xian-Ping ${ }^{1,2, *}$ \\ ${ }^{1}$ Industrial Crops Research Institute, Yunnan Academy of Agricultural Science, Kunming 650205, Yunnan, China; ${ }^{2}$ Scientific Observing and Experi- \\ mental Station of Potato and Rapeseed in Yunnan-Guizhou Plateau, Kunming 650200, Yunnan, China
}

\begin{abstract}
The mining of broad-spectrum resistance genes is the basis of the breeding of potato varieties with high late blight resistance. In this study, 288 samples of late disease resistance selected by the International Potato Center (CIP) for years were planted and were investigated for two consecutive years, which could provide some theoretical and material basis for breeding of late blight resistant varieties and help to study on disease resistance mechanism. The late blight resistance of each sample was evaluated using AUDPC and sAUDPC values. The population simplified genome sequencing was performed by SLAF-seq and carried out by genome-wide association analysis in order to discover the genetic loci and candidate genes related to late blight resistance. There were wide genetic differences in resistance to late blight among 288 samples. Through genome-wide association analysis, based on five analytical models, a total of 82 loci significantly correlated with late blight resistance were identified. Combined with genomic annotation, there were 54 candidate genes known or likely to be associated with late blight resistance in the association interval. Among them, 23 genes were resistant genes, including late blight resistance gene $R 1$ homolog, $S w-5$ homolog $(R 8)$ and Rpi-vnt1, as well as gene encoding pleiotropic resistance protein. Five genes encoded MAPK protein and WRKY transcription factor; one gene was involved in the JA pathway; three genes were associated with salicylic acid pathway; six genes were related to the course of the disease; three genes were involved in the synthesis of phenylpropionic acid. Other genes related
\end{abstract}

\footnotetext{
本研究由国家自然科学基金项目(31760409，31460368), 云南省农业联合重点项目[2017FG001(-012)]和国际马铃薯中心合作项目 TON (14.1432.5-001.00)资助。

This study was supported by the National Natural Science Foundation of China (31760409, 31460368), the Yunnan Agricultural Foundation Projects [2017FG001 (-012)], and the International Potato Center Collaboration Program TON (14.1432.5-001.00).

*通信作者(Corresponding authors): 隋启君, E-mail: 1073480661@qq.com; 李先平, E-mail: 1xianping@hotmail.com

** 同等贡献(Contributed equally to this work)

第一作者联系方式: 蒋伟, E-mail: jiangweiyaas@hotmail.com
}

Received (收稿日期): 2020-04-30; Accepted (接受日期): 2020-09-13; Published online (网络出版日期): 2020-09-28.

URL: https://kns.cnki.net/kcms/detail/11.1809.S.20200927.1805.004.html 
to resistance to late blight included HMGR gene (2) and cytochrome P450 (21).

Keywords: potato; late blight resistance; genome-wide association analysis; resistance gene; CIP

晚疫病是马铃薯的主要病害, 每年可造成我国 马铃薯减产约 $10 \%$ 15\%, 经济损失高达 20 亿美元。 西南地区低温高湿的气候环境，晚疫病发生更严重， 马铃薯减产可达 $15 \% \sim 40 \%{ }^{[1-2]}$ 。提高品种晚疫病抗 性是马铃薯育种重要目标之一，而挖掘持久、高抗 的晚疫病抗性基因对马铃薯抗病育种具有重要的 意义。

20 世纪 50 年代, 育种家将野生种 Solanum demissum 的抗性基因导入到栽培种中，但抗性很快被 新出现的晚疫病生理小种克服 ${ }^{[3-4]}$ 。目前已经鉴定并 克隆到一些对晚疫病表现出广谱抗性的 $R$ 基因。通 过构建 BAC 文库和长片段 PCR 扩增的方法, 从野生 种 S. bulbocastanum 中克隆了第 1 个马铃薯晚疫病广

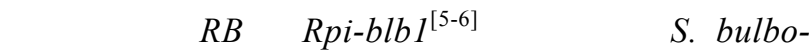
castanum 作图群体，利用 AFLP 分子标记，克隆到另 1 个广谱抗性基因 Rpi-blb2 ${ }^{[4]}$ 。利用野生种 S. venturii 的 5 个作图群体, 结合分离群体分组分析法(bulked segregant analysis，BSA)找到与抗性基因连锁的 AFLP 分子标记, 通过构建 BAC 文库从而克隆了广 谱抗性基因 $R p i-v n t 1^{[7]}$ 。对含有广谱抗性基因 $R 8$ 分 子标记或 QTL 位点的群体构建 BAC 文库, 通过酶切 PCR 克隆到了该基因 ${ }^{[8-9]}$ 。上述这些基因的鉴定主要 是通过构建遗传群体、分子标记定位获得，而通过 自然群体全基因组关联分析定位晚疫病抗性基因的 研究较少。

本研究选择的自然群体引自国际马铃薯中心, 该中心从 19 世纪 80 年代起以淘汰马铃薯晚疫病垂 直抗性而保留其水平抗性为目的, 篮选了一批群体 材料。因此, 该群体中很可能保留着晚疫病广谱抗 性基因。通过对四倍体马铃薯群体连续 2 年的田间 晚疫病抗性评价, 结合群体简化基因组测序获得大 量的 SNP 位点, 进行全基因组关联分析挖掘晚疫病 抗性相关的遗传位点和候选基因, 旨在为晚疫病抗 性品种选育和抗病机理研究提供一定的理论和材料 基础。

\section{1 材料与方法}

\section{1 材料}

引自国际马铃薯中心的 288 份四倍体马铃薯晚疫 病抗性群体材料(表 1), 感病对照为 Desiree (D214)。群 体 A 中晚疫病抗性来源为改良的 S. demissum 行生抗
性, 包括来自安第斯栽培种 S. tuberosum groups andigena、phureja 和 stenotomum, 以及野生种 $S$. acaule 和 S. bulbocastanum 抗性的材料 8 份; 群体 B3 来源于群体 A, 但篮选保留表现出晚疫病水平抗 性的资源材料 82 份; 群体 B1 来自 S. tuberosum groups andigena 的材料 15 份; 群体 LTVR 具有马铃 薯病毒病(PVY、PVX 和 PLRV)抗性、生长期短和适 应温暖环境特性的材料 111 份; 群体 B3-HT 结合群 体 B3 的晚疫病抗性、北美和欧洲品种的耐热性以及 群体 LTVR 特性的材料 34 份; 群体 B3-LTVR 包括 群体 B3 和群体 LTVR 的杂交后代 22 份; 群体 BW 具有细菌性枯萎病抗性的材料 12 份; 群体 PREBRED 具有从野生种导入到四倍体 B3 或 LTVR 中的晚疫病抗性材料 1 份; VARIETY 表示马铃薯品 种 3 份 ${ }^{[10]}$ 。

\section{2 田间试验和表型数据分析}

2015-2016 年，群体材料种植于云南省曲靖市 会泽县试验基地 $\left(26^{\circ} 06^{\prime} \mathrm{N}, 103^{\circ} 22^{\prime} \mathrm{E}\right)$, 海拔 $2650 \mathrm{~m}$ 。 每个材料种植 1 行 10 株, 行距为 $0.7 \mathrm{~m}$, 株距为 0.3 $\mathrm{m}$, 种植 3 次重复。采用 Alpha 试验设计(CIP 提供软 件)，整个生长季进行正常中耕、施肥管理等，但不 喷洒杀菌类农药。

调查田间晚疫病发病比率。发现初始病株, 每 7 $\mathrm{d}$ 调查 1 次, 共调查 8 次, 计算 AUDPC 和 SAUDPC 值。利用 Microsoft Excel 对所观测到的数据进行统 计分析。

$$
\begin{aligned}
& \mathrm{AUDPC}=\sum_{i=1}^{n=1}\left[\frac{\left(x_{i+1}+x_{i}\right)}{2}\right]\left(t_{i+1}-t_{i}\right) \\
& \mathrm{sAUDPC}=\mathrm{AUDPC} \\
& \text { 评估材料 }
\end{aligned}
$$

式中, $n$ 表示总的调查次数, $x_{i}$ 表示第 $i$ 次调查的晚疫 病严重程度, $t_{i}$ 表示第 $i$ 次调查的时间。

\subsection{DNA 提取和测序方法}

利用 CTAB 法提取 DNA，检测合格后利用 Illumina HiSeq2500 测序仪(Illumina, Inc; San Diego, CA, USA)测序。以马铃薯 S. chacoense M6 的基因组 测序信息为参考 ${ }^{[11]}$, 利用北京百迈客生物有限公司 自主研发的简化基因组测序流程 SLAF-seq ${ }^{[12]}$ 进行 电子酶切预测, 根据最佳酶切方案选择 $R s a \mathrm{I}+H a e$ III 进行酶切, 酶切长度在 314 414 的序列被定义为 SLAF 标签, 获得多态性标签 $1,032,778$ 个。以马铃 
薯 S. chacoense M6 为参考基因组, 利用 BWA 软件 ${ }^{[13]}$ 将 reads 比对到参考基因组上, 利用 $\mathrm{GATK}^{[14]}$ 和 SAMtools ${ }^{[15]}$ 软件篮选 SNP。根据完整度大于 0.8 , 次 要基因频率大于 0.05 过滤后, 得到 353,753 个 SNP 位点进行后续的分析。

\section{4 晚疫病抗性全基因组关联分析}

基于 SNP 位点, 利用 TASSEL 软件 ${ }^{[16]}$ 的混合线 性模型(compressed mixed linear model, CMLM), 通 过公式 $y=X \alpha+Q \beta+K \mu+e$ 得到关联值。公式中, 通过 Admixture 软件 ${ }^{[17]}$ 计算样品群体结构 $\mathrm{Q}$ 矩阵, $\mathrm{SPAGeDi}$ 软件 ${ }^{[18]}$ 计算样品间亲缘关系 $\mathrm{K}$ 矩阵, $\mathrm{X}$ 为 基因型矩阵, $y$ 为表型, 最终获得每个 SNP 位点的关 联值，并计算它们的 $P$ 值。以 AUDPC_2015、 AUDPC_2016、sAUDPC_2015、sAUDPC_2016, 以
及这 2 年的 AUDPC 和 sAUDPC 平均值即 AUDPC_Mean 和 AUDPC_Mean 为表型数据, 共分 析 6 个性状。对这些性状利用 GLM、MLM、CMLM、 FASTLMM 和 EMMAX 五种模型进行关联分析。利 用 GGplot2 软件 ${ }^{[19]}$ 绘制 Quantile-Quantile 散点图 (Q-Qplot)，利用 QQman 软件绘制曼哈顿图 (Manhattan), 展示关联分析显著的 SNP 位点。

\section{5 候选基因预测}

对 6 个性状在 5 种模型下获得的显著水平是 0.1 和 0.01 的关联 SNP 位点进行注释。选取这些位点前 后 $100 \mathrm{~kb}$ 范围内的基因, 利用 BLAST 软件分别与 NR、SwissProt、GO、COG 和 KEGG 数据库进行比 对, 获得关联区域内基因注释信息, 并根据注释信 息寻找与晚疫病抗性相关的候选基因。

表 1 自国际马铃薯中心引进的 288 份马铃薯群体材料

Table 1288 potato population resources from CIP

\begin{tabular}{|c|c|c|c|c|c|c|c|c|}
\hline $\begin{array}{l}\text { 编号 } \\
\text { Code }\end{array}$ & $\begin{array}{c}\text { CIP 编号 } \\
\text { CIP accession }\end{array}$ & $\begin{array}{c}\text { 群体 } \\
\text { Population }\end{array}$ & $\begin{array}{l}\text { 编号 } \\
\text { Code }\end{array}$ & $\begin{array}{c}\text { CIP 编号 } \\
\text { CIP accession }\end{array}$ & $\begin{array}{c}\text { 群体 } \\
\text { Population }\end{array}$ & $\begin{array}{l}\text { 编号 } \\
\text { Code }\end{array}$ & $\begin{array}{c}\text { CIP 编号 } \\
\text { CIP accession }\end{array}$ & $\begin{array}{c}\text { 群体 } \\
\text { Population }\end{array}$ \\
\hline $\mathrm{C} 001$ & CIP381381.13 & A & C106 & CIP392740.4 & LTVR & D095 & CIP394900.1 & BW \\
\hline $\mathrm{C} 002$ & CIP391065.69 & B3 & C107 & CIP392745.7 & LTVR & D096 & CIP395011.2 & B3 \\
\hline $\mathrm{C} 003$ & CIP391583.25 & B3 & C108 & CIP392759.1 & LTVR & D097 & CIP395015.6 & B3 \\
\hline $\mathrm{C} 004$ & CIP392617.54 & B3 & C109 & CIP393613.2 & LTVR & D098 & CIP395017.14 & B3 \\
\hline $\mathrm{C} 005$ & CIP392634.52 & B3 & C110 & CIP393615.6 & LTVR & D099 & CIP395017.227 & B3 \\
\hline C006 & CIP393073.179 & B3 & $\mathrm{C} 112$ & CIP397030.31 & LTVR & D100 & CIP395017.229 & B3 \\
\hline $\mathrm{C} 007$ & CIP393073.197 & B3 & $\mathrm{C} 113$ & CIP397035.26 & LTVR & D101 & CIP395017.242 & B3 \\
\hline $\mathrm{C} 008$ & CIP393227.66 & B3 & $\mathrm{C} 114$ & CIP302428.20 & LTVR & D102 & CIP395037.107 & B3 \\
\hline C009 & CIP393228.67 & B3 & $\mathrm{C} 115$ & CIP302476.108 & LTVR & D103 & CIP395077.12 & B3 \\
\hline $\mathrm{C} 010$ & CIP393371.164 & B3 & C116 & CIP302499.30 & LTVR & D105 & CIP395096.2 & B3 \\
\hline $\mathrm{C} 011$ & CIP391004.18 & B3 & C117 & CIP304345.102 & LTVR & D106 & CIP395109.29 & B3 \\
\hline $\mathrm{C} 012$ & CIP392657.171 & B3 & C118 & CIP304350.100 & LTVR & D107 & CIP395109.34 & B3 \\
\hline $\mathrm{C} 013$ & CIP393280.64 & B3 & C119 & CIP304350.118 & LTVR & D108 & CIP395111.13 & B3 \\
\hline $\mathrm{C} 014$ & CIP391047.34 & B3 & $\mathrm{C} 120$ & CIP304350.95 & LTVR & D109 & CIP395112.19 & B3 \\
\hline $\mathrm{C} 015$ & CIP391058.175 & B3 & $\mathrm{C} 121$ & CIP304371.67 & LTVR & D110 & CIP395112.32 & B3 \\
\hline $\mathrm{C} 016$ & CIP393085.5 & B3 & C122 & CIP304383.41 & LTVR & D111 & CIP395112.36 & B3 \\
\hline $\mathrm{C} 017$ & CIP398192.213 & B3-HT & C123 & CIP304383.80 & LTVR & D112 & CIP395112.6 & B3 \\
\hline $\mathrm{C} 018$ & CIP398098.119 & B3-HT & C124 & CIP304387.39 & LTVR & D113 & CIP395123.6 & B3 \\
\hline C019 & CIP398098.203 & B3-HT & C125 & CIP304405.47 & LTVR & D114 & CIP395169.17 & B3 \\
\hline $\mathrm{C} 020$ & CIP398180.253 & B3-HT & C127 & CIP397077.16 & LTVR & D117 & CIP395448.1 & BW \\
\hline $\mathrm{C} 021$ & CIP398180.289 & B3-HT & C128 & CIP391919.3 & BW & D118 & CIP396004.225 & B3 \\
\hline $\mathrm{C} 024$ & CIP398193.553 & B3-HT & C129 & CIP391930.1 & BW & D119 & CIP396004.263 & B3 \\
\hline $\mathrm{C} 025$ & CIP398203.509 & B3-HT & C130 & CIP391931.1 & BW & D121 & CIP396008.104 & B3 \\
\hline $\mathrm{C} 026$ & CIP398208.219 & B3-HT & C131 & CIP394906.6 & BW & D123 & CIP396009.258 & B3 \\
\hline $\mathrm{C} 027$ & CIP398208.33 & B3-HT & C132 & CIP395438.1 & BW & D124 & CIP396012.266 & B3 \\
\hline $\mathrm{C} 028$ & CIP398208.58 & B3-HT & C133 & CIP394904.20 & BW & D126 & CIP396023.109 & B3 \\
\hline C029 & CIP398208.704 & B3-HT & D001 & CIP300055.32 & LTVR & D127 & CIP396026.101 & B3 \\
\hline
\end{tabular}


(续表 1)

\begin{tabular}{|c|c|c|c|c|c|c|c|c|}
\hline $\begin{array}{l}\text { 编号 } \\
\text { Code }\end{array}$ & $\begin{array}{c}\text { CIP 编号 } \\
\text { CIP accession }\end{array}$ & $\begin{array}{c}\text { 群体 } \\
\text { Population }\end{array}$ & $\begin{array}{l}\text { 编号 } \\
\text { Code }\end{array}$ & $\begin{array}{c}\text { CIP 编号 } \\
\text { CIP accession }\end{array}$ & $\begin{array}{c}\text { 群体 } \\
\text { Population }\end{array}$ & $\begin{array}{l}\text { 编号 } \\
\text { Code }\end{array}$ & $\begin{array}{c}\text { CIP 编号 } \\
\text { CIP accession }\end{array}$ & $\begin{array}{c}\text { 群体 } \\
\text { Population }\end{array}$ \\
\hline $\mathrm{C} 030$ & CIP301024.14 & B3-LTVR & D002 & CIP300063.9 & LTVR & D128 & CIP396026.103 & B3 \\
\hline $\mathrm{C} 031$ & CIP301029.18 & B3-LTVR & D003 & CIP300065.4 & LTVR & D130 & CIP396031.108 & B3 \\
\hline $\mathrm{C} 032$ & CIP301040.63 & B3-LTVR & D005 & CIP300135.3 & LTVR & D131 & CIP396031.119 & B3 \\
\hline $\mathrm{C} 033$ & CIP300046.22 & LTVR & D007 & CIP301023.15 & B3-LTVR & D132 & CIP396033.102 & B3 \\
\hline $\mathrm{C} 034$ & CIP300048.12 & LTVR & D008 & CIP301024.95 & B3-LTVR & D133 & CIP396034.103 & B3 \\
\hline $\mathrm{C} 035$ & CIP300054.29 & LTVR & D009 & CIP301026.23 & B3-LTVR & D134 & CIP396034.268 & B3 \\
\hline C036 & CIP300056.33 & LTVR & D011 & CIP301041.26 & B3-LTVR & D137 & CIP396038.101 & B3 \\
\hline $\mathrm{C} 037$ & CIP300063.4 & LTVR & D012 & CIP301044.36 & B3-LTVR & D138 & CIP396038.105 & B3 \\
\hline $\mathrm{C} 038$ & CIP300066.11 & LTVR & D013 & CIP301045.74 & B3-LTVR & D139 & CIP396038.107 & B3 \\
\hline C039 & CIP300072.1 & LTVR & D014 & CIP301055.53 & B3-LTVR & D140 & CIP396043.226 & B3 \\
\hline $\mathrm{C} 040$ & CIP300093.14 & LTVR & D015 & CIP301056.54 & B3-LTVR & D142 & CIP396063.1 & B3-LTVR \\
\hline $\mathrm{C} 041$ & CIP300099.22 & LTVR & D017 & CIP303381.106 & LTVR & D143 & CIP396063.16 & B3-LTVR \\
\hline $\mathrm{C} 042$ & CIP300101.11 & LTVR & D018 & CIP303381.30 & LTVR & D144 & CIP396180.22 & B3-LTVR \\
\hline $\mathrm{C} 043$ & CIP379706.27 & LTVR & D019 & CIP304347.6 & LTVR & D145 & CIP396240.2 & B3 \\
\hline C044 & CIP385499.11 & LTVR & D021 & CIP304350.18 & LTVR & D147 & CIP396241.4 & B3 \\
\hline $\mathrm{C} 046$ & CIP388676.1 & LTVR & D022 & CIP304350.78 & LTVR & D148 & CIP396244.12 & B3 \\
\hline $\mathrm{C} 047$ & CIP388972.22 & LTVR & D023 & CIP304351.109 & LTVR & D149 & CIP396268.1 & B3-LTVR \\
\hline $\mathrm{C} 048$ & CIP390478.9 & LTVR & D024 & CIP304351.31 & LTVR & D150 & CIP396268.9 & B3-LTVR \\
\hline C049 & CIP391207.2 & LTVR & D025 & CIP304366.46 & LTVR & D151 & CIP396269.14 & B3-LTVR \\
\hline $\mathrm{C} 050$ & CIP391382.18 & LTVR & D028 & CIP304371.58 & LTVR & D152 & CIP396269.16 & B3-LTVR \\
\hline $\mathrm{C} 051$ & CIP392781.1 & LTVR & D029 & CIP304387.17 & LTVR & D154 & CIP396272.18 & B3-LTVR \\
\hline $\mathrm{C} 052$ & CIP392797.22 & LTVR & D030 & CIP304387.92 & LTVR & D156 & CIP396272.21 & B3-LTVR \\
\hline $\mathrm{C} 053$ & CIP392822.3 & LTVR & D031 & CIP304394.56 & LTVR & D157 & CIP396272.37 & B3-LTVR \\
\hline $\mathrm{C} 054$ & CIP392973.48 & LTVR & D033 & CIP304399.5 & LTVR & D158 & CIP396273.48 & B3-LTVR \\
\hline $\mathrm{C} 056$ & CIP394034.65 & LTVR & D035 & CIP304406.31 & LTVR & D160 & CIP397006.18 & LTVR \\
\hline $\mathrm{C} 057$ & CIP394034.7 & LTVR & D036 & CIP374080.5 & A & D162 & CIP397039.53 & LTVR \\
\hline $\mathrm{C} 058$ & CIP394579.36 & LTVR & D037 & CIP377744.1 & A & D164 & CIP397060.19 & LTVR \\
\hline $\mathrm{C} 059$ & CIP394600.52 & LTVR & D040 & CIP381178.14 & A & D165 & CIP398098.205 & B3-HT \\
\hline $\mathrm{C} 061$ & CIP394613.139 & LTVR & D041 & CIP381379.12 & A & D166 & CIP398098.231 & B3-HT \\
\hline $\mathrm{C} 062$ & CIP394613.32 & LTVR & D042 & CIP381381.9 & A & D167 & CIP398098.570 & B3-HT \\
\hline $\mathrm{C} 063$ & CIP394614.117 & LTVR & D043 & CIP381403.16 & A & D168 & CIP398098.65 & B3-HT \\
\hline C064 & CIP394881.8 & LTVR & D044 & CIP384321.3 & A & D169 & CIP398180.144 & B3-HT \\
\hline $\mathrm{C} 065$ & CIP395186.6 & LTVR & D047 & CIP387164.4 & B3 & D170 & CIP398190.112 & B3-HT \\
\hline $\mathrm{C} 066$ & CIP395193.6 & LTVR & D048 & CIP389746.2 & B3 & D171 & CIP398190.200 & B3-HT \\
\hline $\mathrm{C} 067$ & CIP395195.7 & LTVR & D050 & CIP391011.17 & B3 & D172 & CIP398190.404 & B3-HT \\
\hline $\mathrm{C} 068$ & CIP395196.4 & LTVR & D051 & CIP391046.14 & B3 & D173 & CIP398190.523 & B3-HT \\
\hline $\mathrm{C} 069$ & CIP395197.5 & LTVR & D052 & CIP391065.81 & B3 & D174 & CIP398190.530 & B3-HT \\
\hline $\mathrm{C} 070$ & CIP395432.51 & LTVR & D053 & CIP391580.30 & B3 & D175 & CIP398190.571 & B3-HT \\
\hline $\mathrm{C} 071$ & CIP395434.1 & LTVR & D054 & CIP391585.179 & B3 & D176 & CIP398190.605 & B3-HT \\
\hline $\mathrm{C} 072$ & CIP395436.8 & LTVR & D055 & CIP391585.5 & B3 & D177 & CIP398190.615 & B3-HT \\
\hline $\mathrm{C} 076$ & CIP397012.22 & LTVR & D057 & CIP392025.7 & LTVR & D178 & CIP398190.735 & B3-HT \\
\hline $\mathrm{C} 078$ & CIP397016.7 & LTVR & D058 & CIP392285.72 & BW & D179 & CIP398192.41 & B3-HT \\
\hline C079 & CIP397029.21 & LTVR & D059 & CIP392633.64 & B3 & D180 & CIP398192.553 & B3-HT \\
\hline $\mathrm{C} 080$ & CIP397036.7 & LTVR & D060 & CIP392634.49 & B3 & D181 & CIP398192.592 & B3-HT \\
\hline $\mathrm{C} 081$ & CIP397039.51 & LTVR & D061 & CIP392637.10 & B3 & D182 & CIP398193.158 & B3-HT \\
\hline
\end{tabular}


(续表 1)

\begin{tabular}{|c|c|c|c|c|c|c|c|c|}
\hline $\begin{array}{l}\text { 编号 } \\
\text { Code }\end{array}$ & $\begin{array}{c}\text { CIP 编号 } \\
\text { CIP accession }\end{array}$ & $\begin{array}{c}\text { 群体 } \\
\text { Population } \\
\end{array}$ & $\begin{array}{l}\text { 编号 } \\
\text { Code }\end{array}$ & $\begin{array}{c}\text { CIP 编号 } \\
\text { CIP accession }\end{array}$ & $\begin{array}{c}\text { 群体 } \\
\text { Population } \\
\end{array}$ & $\begin{array}{l}\text { 编号 } \\
\text { Code }\end{array}$ & $\begin{array}{c}\text { CIP 编号 } \\
\text { CIP accession }\end{array}$ & $\begin{array}{c}\text { 群体 } \\
\text { Population } \\
\end{array}$ \\
\hline $\mathrm{C} 082$ & CIP397044.25 & LTVR & D064 & CIP392821.1 & LTVR & D183 & CIP398193.650 & B3-HT \\
\hline $\mathrm{C} 083$ & CIP397055.2 & LTVR & D065 & CIP393075.54 & B3 & D185 & CIP398208.29 & B3-HT \\
\hline C084 & CIP397065.2 & LTVR & D067 & CIP393077.54 & B3 & D186 & CIP398208.505 & B3-HT \\
\hline $\mathrm{C} 085$ & CIP397067.2 & LTVR & D068 & CIP393079.24 & B3 & D187 & CIP398208.620 & B3-HT \\
\hline C086 & CIP397069.5 & LTVR & D069 & CIP393079.4 & B3 & D188 & CIP398208.670 & B3-HT \\
\hline $\mathrm{C} 087$ & CIP397073.15 & LTVR & D070 & CIP393083.2 & B3 & D189 & CIP399001.44 & B1 \\
\hline $\mathrm{C} 088$ & CIP397078.12 & LTVR & D071 & CIP393084.31 & B3 & D190 & CIP399004.19 & B1 \\
\hline C089 & CIP397079.26 & LTVR & D072 & CIP393220.54 & B3 & D192 & CIP399049.14 & B1 \\
\hline $\mathrm{C} 090$ & CIP397079.6 & LTVR & D074 & CIP393248.55 & B3 & D193 & CIP399049.16 & B1 \\
\hline C091 & CIP397098.12 & LTVR & D075 & CIP393280.57 & B3 & D194 & CIP399049.22 & B1 \\
\hline C092 & CIP397099.6 & LTVR & D077 & CIP393284.39 & B3 & D195 & CIP399053.11 & B1 \\
\hline $\mathrm{C} 093$ & CIP397100.9 & LTVR & D078 & CIP393339.242 & B3 & D196 & CIP399053.15 & B1 \\
\hline C094 & CIP397196.3 & LTVR & D079 & CIP393349.68 & B3 & D199 & CIP399067.22 & B1 \\
\hline $\mathrm{C} 095$ & CIP397196.8 & LTVR & D080 & CIP393371.157 & B3 & D201 & CIP399072.28 & B1 \\
\hline C096 & CIP397197.9 & LTVR & D081 & CIP393371.159 & B3 & D203 & CIP399075.32 & B1 \\
\hline C097 & CIP398014.2 & LTVR & D082 & CIP393371.58 & B3 & D205 & CIP399078.11 & B1 \\
\hline C098 & CIP388611.22 & LTVR & D083 & CIP393382.44 & B3 & D206 & CIP399079.22 & B1 \\
\hline C099 & CIP388615.22 & LTVR & D084 & CIP393385.39 & B3 & D207 & CIP399083.4 & B1 \\
\hline $\mathrm{C} 100$ & CIP389468.3 & LTVR & D085 & CIP393385.47 & B3 & D209 & CIP399085.23 & B1 \\
\hline $\mathrm{C} 101$ & CIP390637.1 & LTVR & D088 & CIP393617.1 & LTVR & D210 & CIP399085.30 & B1 \\
\hline $\mathrm{C} 102$ & CIP391180.6 & LTVR & D089 & CIP394223.19 & LTVR & D212 & CIP694474.33 & PREBRED \\
\hline $\mathrm{C} 103$ & CIP391533.1 & LTVR & D092 & CIP394895.7 & BW & D214 & CIP800048 & VARIETY \\
\hline C104 & CIP391724.1 & LTVR & D093 & CIP394898.13 & BW & D216 & CIP800923 & VARIETY \\
\hline $\mathrm{C} 105$ & CIP392032.2 & LTVR & D094 & CIP394899.5 & BW & D217 & CIP800959 & VARIETY \\
\hline
\end{tabular}

群体 A 中晚疫病抗性来源为改良的 S. demissum 行生抗性; 群体 B3 来源于群体 A, 篮选保留表现出晚疫病水平抗性的资源; 群体 B1 来自 S. tuberosum groups andigena 的抗性材料; 群体 LTVR 具有马铃薯病毒病(PVY、PVX 和 PLRV)抗性、生长期短和适应温暖环境 特性的材料; 群体 B3-HT 结合群体 B3 的晚疫病抗性、北美和欧洲品种的耐热性以及群体 LTVR 特性的材料; 群体 B3-LTVR 包括群 体 B3 和群体 LTVR 的杂交后代; 群体 BW 具有细菌性枯萎病抗性的材料; 群体 PREBRED 具有从野生种导入到四倍体 B3 或 LTVR 中的晚疫病抗性材料; VARIETY 表示马铃薯品种。

The late blight resistance of population A was resource from S. demissum-derived; population B3 was derived from population A, and resources showing late blight resistance were screened and retained; population B1 was derived from the resistant material of S. tuberosum groups andigena; population LTVR were resistant to potato virus disease (PVY, PVX, and PLRV), and had a short growth period and adapted to warm climate; the population B3-HT included late blight resistance from population B3, the heat resistance of North American and European varieties, and the properties of population LTRV; population B3-LTVR included hybrid offsprings of population B3 and LTVR; population BW had bacterial wilt resistance; the PREBRED had late blight resistance imported from wild species to tetraploid B3 or LTVR; VARIETY represented potato varieties.

\section{2 结果与分析}

\section{1 晚疫病抗性的表型分析}

通过对 288 份马铃薯资源材料连续 2 年的晚疫 病田间抗性评价发现, 晚疫病抗性评价值 AUDPC 和 sAUDPC 呈连续分布。2015 年所有材料的 AUDPC 平均值为 2244.1, 范围是 121.3 4740.8, 变 异系数为 $61.73 \%, \mathrm{sAUDPC}$ 平均值为 3.9 , 范围是 $0.2 \sim 8.3$, 变异系数为 $61.56 \% ; 2016$ 年所有材料的
AUDPC 平均值为 1228.5, 范围是 72.7 3371.3, 变异系 数为 $80.47 \%$, sAUDPC 平均值为 3.6, 范围是 0.2 9.5, 变异系数为 $80.21 \%$ 。表明该群体表现出晚疫病抗性广 泛的遗传差异, 且受多基因控制, 属于数量性状遗传。 相关性分析中, 2015-2016 年 AUDPC 值相关系数为 0.8988 ，高度线性相关; sAUDPC 值相关系数为 0.8974 , 高度线性相关(图 1)。表明 2 年间群体材料田间晚疫病 抗性表现一致, 抗性稳定。 

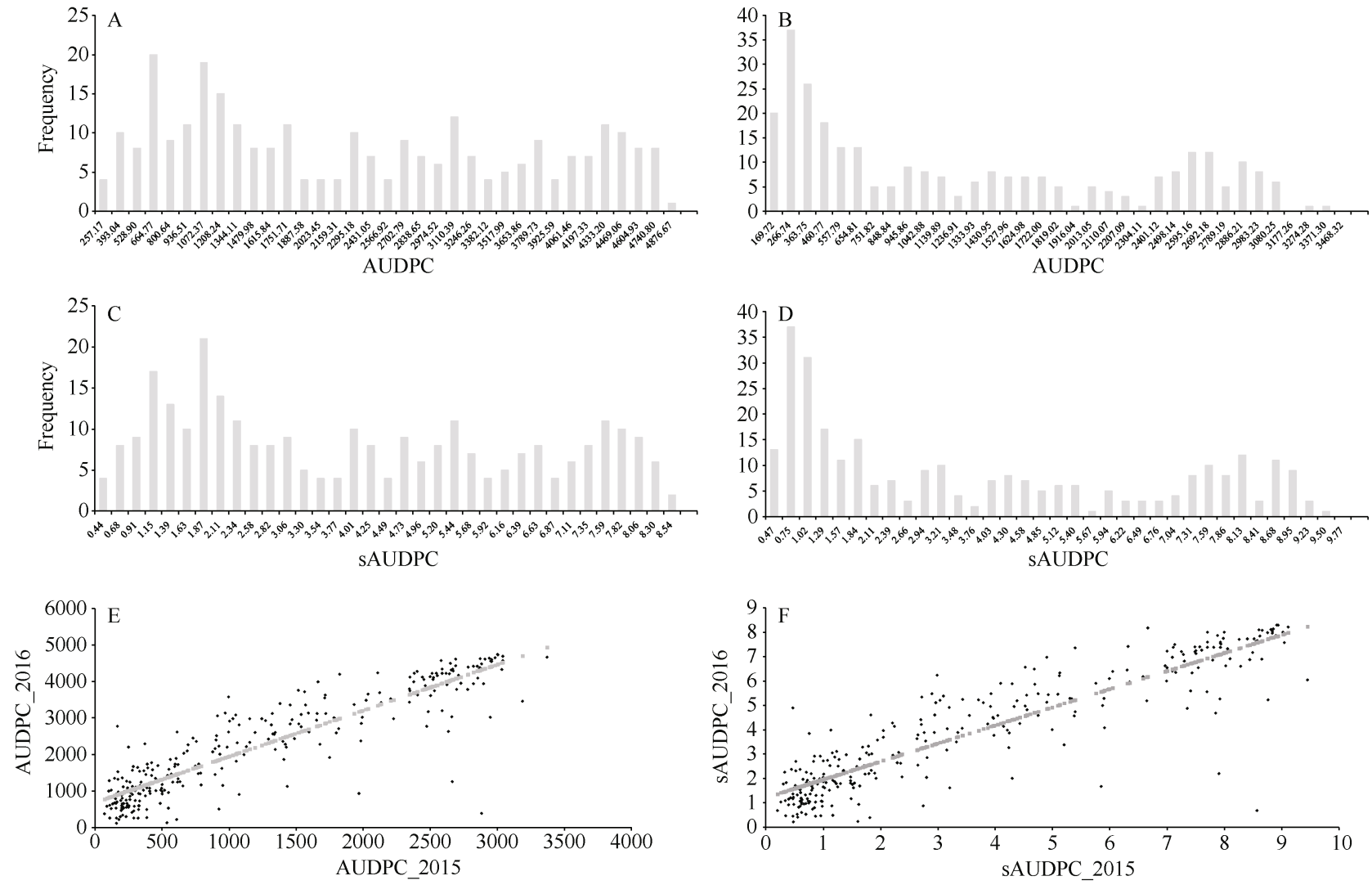

图 1288 份群体材料 AUDPC 和 SAUDPC 频次分布图及相关性散图(2015-2016 年)

Fig. 1 Frequency distribution and correlation of the AUDPC and SAUDPC values of 288 samples in 2015 and 2016

A: 2015 年 AUDPC 值; B: 2016 年 AUDPC 值; C: 2015 年 sAUDPC 值; D: 2016 年 sAUDPC 值; E: 2015 年和 2016 年 AUDPC 值相关性 散布图; F: 2015 年和 2016 年 sAUDPC 值相关性散布图。

A: AUDPC value in 2015; B: AUDPC value in 2016; C: sAUDPC in 2015; D: sAUDPC in 2016; E: correlation plots of AUDPC values between 2015 and 2016; F: correlation plots of sAUDPC values between 2015 and 2016.

\section{2 马铃薯晚疫病抗性的全基因组关联分析}

5 种模型下对 6 个性状分析的 QQ 图结果表明, 在所有模型下关联到显著相关的 SNP 位点都是可 靠的(图 2 是 2 个性状 AUDPC_mean 和 sAUDPC_mean 在 5 种模型下的 QQ 图)。在 5 种分析模型下，共 关联到与 6 种晚疫病抗性性状显著相关(显著水平 为 0.1 和 0.01)的 SNP 位点 82 个(表 2 和图 3)。其 中, 6 个 SNP 位点分布在 3 号染色体(图 3-A, B); 3 个 SNP 位点分布在 4 号染色体(图 3-A，B); 1 个 SNP 位点分布在 5 号染色体(图 3-B, C); 分别有 2 个 SNP 位点分布在 6 号、7 号染色体(图 3-A, B, D); 66 个 SNP 位点分布在 9 号染色体(图 3-A D); 各 有 1 个 SNP 位点分布在 11 号和 12 号染色体(图 3-A, $\mathrm{B}, \mathrm{D})$ 。在 82 个 SNP 位点中, 20 个 SNP 位点在所 有模型分析中, 2 种显著水平情况下，与所有性状 都相关。它们是 $31,375,673$ (第 9 个)、 $31,707,832$ (第 23 个)、32,382,888 (第 25 个)、32,973,114 (第 35 个)、33,118,463 (第 36 个)、33,145,927 (第 38
个)、33,148,740 (第 39 个)、 $33,156,262$ (第 40 个)、 $33,211,574$ (第 43 个)、33,244,698 (第 48 个)、 $33,283,731$ (第 49 个)、33,283,949 (第 50 个)、 $33,451,096$ (第 53 个)、33,480,937 (第 55 个)、 $34,959,324$ (第 62 个)、35,060,347 (第 66 个)、 $35,065,836$ (第 67 个)、35,123,523 (第 72 个)、 $35,212,534$ (第 77 个)、35,256,410 (第 80 个)。同一 性状下, GLM 模型分析定位到的 SNP 位点数目最多, 而 MLM 模型分析定位到的 SNP 位点数目最少。在 GLM 模型下分析, 显著水平为 0.1 时定位到与 AUDPC_Mean 相关的 SNP 位点数目 (74 个)最多(表 2)。

\section{3 候选基因预测}

本研究在 82 个关联 SNP 位点上下游区域 $100 \mathrm{~kb}$, 共检测到 922 个候选基因。其中, 460 个候选基因在 所有分析(60 种)中都存在, 其余基因在 2 56 种分析 中出现。根据基因组注释, 篮选出 54 个已知或可能 与晚疫病抗性相关的基因(表 3)。其中, 23 个基因为 抗性基因包括晚疫病抗性基因 $R 1$ 同源基因、 $S w-5$ 

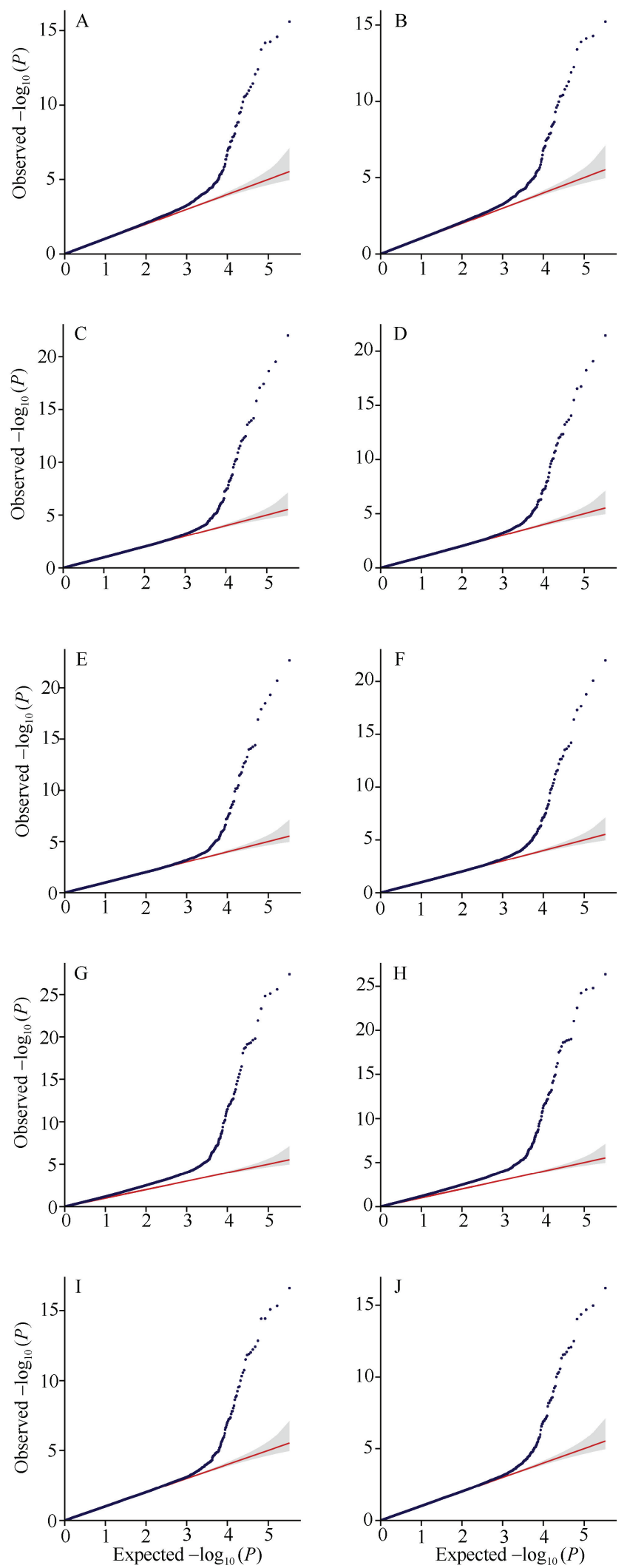

图 2 AUDPC_mean (A: CMLM; C: EMMAX; E: FASTLMM; G: GLM; I: MLM 模型)和 sAUDPC_mean (B: CMLM; D: EMMAX; F: FASTLMM; H: GLM; J: MLM 模型)不同模型下的 QQ 图

Fig. 2 QQ plots of AUDPC_mean and sAUDPC_mean under different analytical model (AUDPC A: CMLM; C: EMMAX; E: FASTLMM; G: GLM; I: MLM; SAUDPC B: CMLM; D: EMMAX; F: FASTLMM; H: GLM; J: MLM) 
表 26 个马铃薯晚疫病抗性性状在不同分析模型和显著水平下的显著关联标记

Table 2 Significant correlation markers of six phenotype of potato resistance to late blight under different analytical models and significant levels

\begin{tabular}{|c|c|c|c|}
\hline 编号 & SNP 位点 & 染色体 & 不同模型、显著水平下与 SNP 相关联的性状 \\
\hline Number & SNP locus & Chr. & Traits correlated with SNP under different analytical models and significant level \\
\hline 1 & 10774833 & 3 & $5^{\mathrm{d}}-0.1,6_{-}^{\mathrm{d}} 0.1$ \\
\hline 2 & 10774840 & 3 & $5^{\mathrm{d}}-0.1$ \\
\hline 3 & 10774841 & 3 & $5^{\mathrm{d}}-0.1$ \\
\hline 4 & 14767856 & 3 & $3^{\mathrm{d}, \mathrm{e}}-0.1,4^{\mathrm{d}, \mathrm{e}}-0.1,6^{\mathrm{d}, \mathrm{e}}-0.1$ \\
\hline 5 & 18132045 & 3 & $3^{\mathrm{d}}{ }_{-} 0.1,4_{-}^{\mathrm{d}}{ }_{-}^{0.1}$ \\
\hline 6 & 32015964 & 3 & $3^{\mathrm{d}}-0.1,4^{\mathrm{d}}-0.1,6^{\mathrm{d}}-0.1$ \\
\hline 7 & 7144093 & 4 & $5^{\mathrm{d}} 0.1,6_{-}^{\mathrm{d}} 0.1$ \\
\hline 8 & 17868575 & 4 & $3^{\mathrm{d}}-0.1,4_{-}^{\mathrm{d}} 0.1$ \\
\hline 9 & 31375673 & 4 & 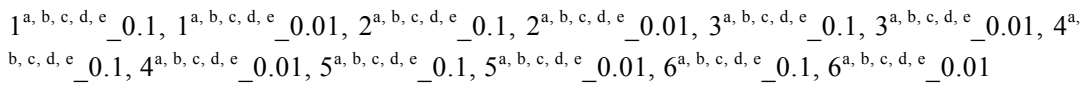 \\
\hline 10 & 4579130 & 5 & $3^{\mathrm{b}, \mathrm{c}, \mathrm{d}}-0.1,3^{\mathrm{b}, \mathrm{c}}{ }_{-} 0.01,4^{\mathrm{b}, \mathrm{c}, \mathrm{d}}-0.1,4^{\mathrm{b}}{ }_{-} 0.01$ \\
\hline 11 & 2241058 & 6 & 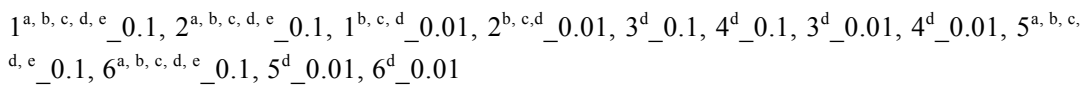 \\
\hline 12 & 2241060 & 6 & 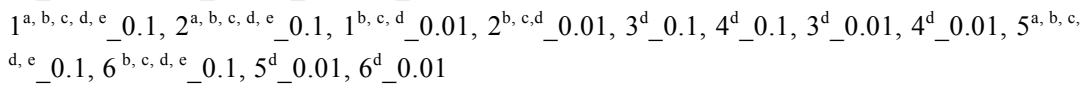 \\
\hline 13 & 17610976 & 7 & $1_{-}^{\mathrm{d}} 0.1,2^{\mathrm{d}} \__{-} .1,5^{\mathrm{d}}-0.1,6^{\mathrm{d}}{ }_{-} 0.1,5^{\mathrm{d}}{ }_{-} 0.01$ \\
\hline 14 & 37485429 & 7 & $1_{-}^{\mathrm{d}} 0.1,2^{\mathrm{d}}-0.1,5^{\mathrm{d}}-0.1,6^{\mathrm{d}}-0.1$ \\
\hline 15 & 22968094 & 9 & $1^{\mathrm{d}}-_{0.1}, 2^{\mathrm{d}}{ }_{-} 0.1,5^{\mathrm{d}}-0.1,6^{\mathrm{d}}{ }_{-} 0.1$ \\
\hline 16 & 30491004 & 9 & $\begin{array}{l}1_{-}^{\mathrm{d}} 0.1,1^{\mathrm{d}}{ }_{-} 0.01,2^{\mathrm{d}}-0.1,2^{\mathrm{d}}-0.01,3^{\mathrm{d}}-0.1,3^{\mathrm{d}}{ }_{-}^{\mathrm{d}} 0.01,4^{\mathrm{d}}{ }_{-} 0.1,4_{-}^{\mathrm{d}}-0.01,5^{\mathrm{d}}-0.1,5^{\mathrm{d}}-0.01,6^{\mathrm{d}}-0.1, \\
6^{\mathrm{d}} 0.01\end{array}$ \\
\hline 17 & 30518418 & 9 & $\begin{array}{l}1^{\mathrm{d}, \mathrm{e}}-0.1,1^{\mathrm{d}}-0.01,2^{\mathrm{d}, \mathrm{e}}-0.1,2^{\mathrm{d}}-0.01,3^{\mathrm{d}}-0.1,3^{\mathrm{d}}-0.01,4^{\mathrm{d}}-0.1,4^{\mathrm{d}}-0.01,5^{\mathrm{d}, \mathrm{e}}-0.1,5^{\mathrm{d}}-0.01,6^{\mathrm{d},} \\
{ }^{\mathrm{e}} 0.1,6^{\mathrm{d}}{ }_{-} 0.01\end{array}$ \\
\hline 18 & 30522602 & 9 & $\begin{array}{l}1^{\mathrm{d}}-0.1,1^{\mathrm{d}}-0.01,2^{\mathrm{d}}-0.1,2^{\mathrm{d}}-0.01,3^{\mathrm{d}}-0.1,3^{\mathrm{d}}-0.01,4^{\mathrm{d}}-0.1,4^{\mathrm{d}}-0.01,5^{\mathrm{d}, \mathrm{e}}-0.1,5^{\mathrm{d}}-0.01,6^{\mathrm{d},} \\
{ }^{\mathrm{e}} 0.1,6^{\mathrm{d}}{ }^{\mathrm{d}} 0.01\end{array}$ \\
\hline 19 & 30542106 & 9 & $\begin{array}{l}1_{-}^{\mathrm{d}} 0.1,1^{\mathrm{d}}{ }_{-} 0.01,2^{\mathrm{d}}-0.1,2^{\mathrm{d}}-0.01,3^{\mathrm{d}}-0.1,3^{\mathrm{d}}-0.01,4^{\mathrm{d}}{ }_{-} 0.1,4_{-}^{\mathrm{d}}-0.01,5^{\mathrm{d}}-0.1,5^{\mathrm{d}}-0.01,6^{\mathrm{d}}-0.1, \\
6^{\mathrm{d}}-0.01\end{array}$ \\
\hline 20 & 30550212 & 9 & $\begin{array}{l}1^{\mathrm{d}}-0.1,1^{\mathrm{d}}{ }_{-} 0.01,2^{\mathrm{d}}-0.1,2^{\mathrm{d}}-0.01,3^{\mathrm{d}}-0.1,3^{\mathrm{d}}{ }_{-}^{\mathrm{d}} 0.01,4^{\mathrm{d}}{ }_{-} 0.1,4^{\mathrm{d}}{ }_{-} 0.01,5^{\mathrm{d}}-0.1,5^{\mathrm{d}}-0.01,6^{\mathrm{d}}{ }_{-} 0.1, \\
6^{\mathrm{d}}-0.01\end{array}$ \\
\hline 21 & 30568973 & 9 & $\begin{array}{l}1^{\mathrm{d}}-0.1,1^{\mathrm{d}}{ }_{-} 0.01,2^{\mathrm{d}}-0.1,2^{\mathrm{d}}-0.01,3^{\mathrm{d}}-0.1,3^{\mathrm{d}}{ }_{-}^{\mathrm{d}} 0.01,4^{\mathrm{d}}{ }_{-} 0.1,4^{\mathrm{d}}{ }_{-} 0.01,5^{\mathrm{d}}-0.1,5^{\mathrm{d}}-0.01,6^{\mathrm{d}}-0.1, \\
6^{\mathrm{d}}-0.01\end{array}$ \\
\hline 22 & 31001787 & 9 & $\begin{array}{l}1_{-}^{\mathrm{d}} 0.1,1^{\mathrm{d}}-0.01,2^{\mathrm{d}}-0.1,2^{\mathrm{d}}-0.01,3^{\mathrm{d}}-0.1,3^{\mathrm{d}}-0.01,4^{\mathrm{d}}{ }_{-} 0.1,4_{-}^{\mathrm{d}}-0.01,5^{\mathrm{d}}-0.1,5^{\mathrm{d}}-0.01,6^{\mathrm{d}}{ }_{-} 0.1, \\
6^{\mathrm{d}}-0.01\end{array}$ \\
\hline 23 & 31707832 & 9 & 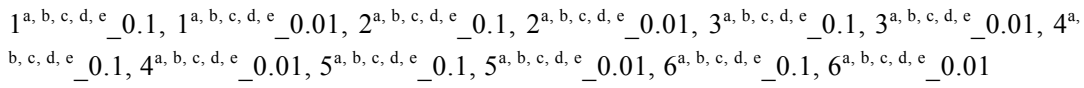 \\
\hline 24 & 32170544 & 9 & $1_{-}^{\mathrm{d}} 0.1,2^{\mathrm{d}}-_{-} 0.1,3^{\mathrm{d}}{ }_{-} 0.1,4_{-}^{\mathrm{d}}-0.1,5^{\mathrm{d}}{ }_{-} 0.1,5^{\mathrm{d}}-0.01,6^{\mathrm{d}}{ }_{-} 0.1,6^{\mathrm{d}}{ }_{-} 0.01$ \\
\hline 25 & 32382888 & 9 & 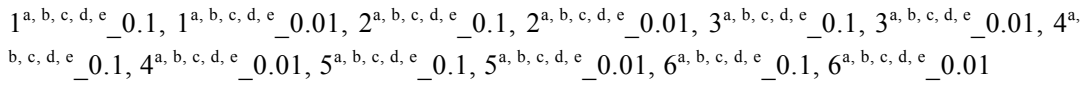 \\
\hline 26 & 32442672 & 9 & $1_{-}^{\mathrm{d}}-0.1$ \\
\hline 27 & 32468577 & 9 & 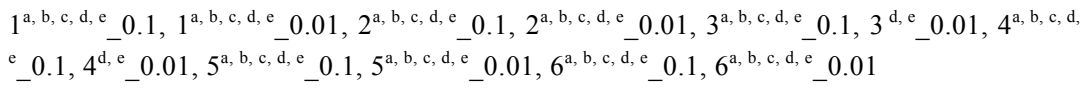 \\
\hline 28 & 32627854 & 9 & $\begin{array}{l}1^{\mathrm{a}, \mathrm{b}, \mathrm{c}, \mathrm{d}, \mathrm{e}}-0.1,1^{\mathrm{a}, \mathrm{b}, \mathrm{c}, \mathrm{d}}-0.01,2^{\mathrm{a}, \mathrm{b}, \mathrm{c}, \mathrm{d}, \mathrm{e}}-0.1,2^{\mathrm{a}, \mathrm{b}, \mathrm{c}, \mathrm{d}}-0.01,3^{\mathrm{b}, \mathrm{c}, \mathrm{d}}-0.1,3^{\mathrm{d}}{ }^{\mathrm{d}} 0.01,4^{\mathrm{b}, \mathrm{c}, \mathrm{d}}{ }^{-} 0.1, \\
4^{\mathrm{d}}-0.01,5^{\mathrm{a}, \mathrm{b}, \mathrm{c}, \mathrm{d}, \mathrm{e}}-0.1,5^{\mathrm{a}, \mathrm{b}, \mathrm{c}, \mathrm{d}}-0.01,6^{\mathrm{a}, \mathrm{b}, \mathrm{c}, \mathrm{d}, \mathrm{e}}-0.1,6^{\mathrm{b}, \mathrm{c}, \mathrm{d}}-0.01\end{array}$ \\
\hline 29 & 32642867 & 9 & 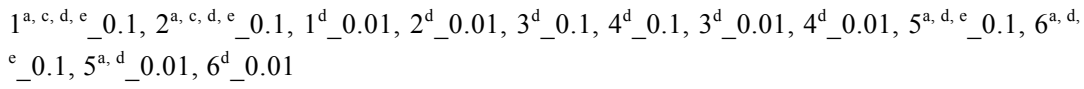 \\
\hline 30 & 32747131 & 9 & 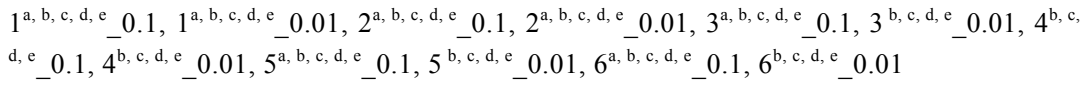 \\
\hline 31 & 32749768 & 9 & $3_{-}^{\mathrm{d}} 0.1,4_{-}^{\mathrm{d}} 0.1$ \\
\hline 32 & 32749770 & 9 & $3^{\mathrm{d}}-0.1,3^{\mathrm{d}}-_{0.01}, 4^{\mathrm{d}}-0.1,4^{\mathrm{d}}-0.01,5^{\mathrm{d}}-0.1,6^{\mathrm{d}}-_{0.1}$ \\
\hline
\end{tabular}


(续表 2)

\begin{tabular}{|c|c|c|c|}
\hline $\begin{array}{c}\text { 编号 } \\
\text { Number }\end{array}$ & $\begin{array}{l}\text { SNP 位点 } \\
\text { SNP locus }\end{array}$ & $\begin{array}{l}\text { 染色体 } \\
\text { Chr. }\end{array}$ & $\begin{array}{l}\text { 不同模型、显著水平下与 SNP 相关联的性状 } \\
\text { Traits correlated with SNP under different analytical models and significant level }\end{array}$ \\
\hline 33 & 32752786 & 9 & 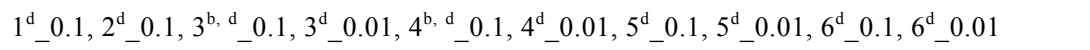 \\
\hline 34 & 32753035 & 9 & $1^{\mathrm{d}}{ }_{-} 0.1,2^{\mathrm{d}}{ }_{-} 0.1,3^{\mathrm{d}}{ }_{-} 0.1,3^{\mathrm{d}}-0.01,4^{\mathrm{d}}-0.1,4^{\mathrm{d}}{ }_{-} 0.01,5^{\mathrm{d}}{ }_{-} 0.1,5^{\mathrm{d}}-0.01,6^{\mathrm{d}}{ }_{-} 0.1,6^{\mathrm{d}}-0.01$ \\
\hline 35 & 32973114 & 9 & 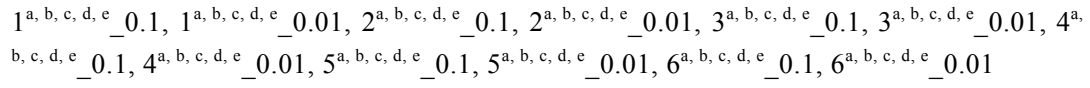 \\
\hline 36 & 33118463 & 9 & 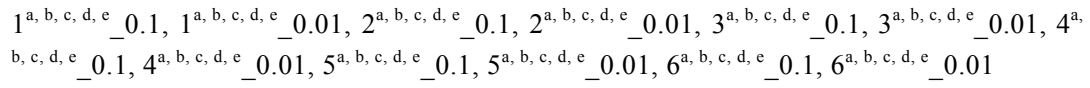 \\
\hline 37 & 33145926 & 9 & 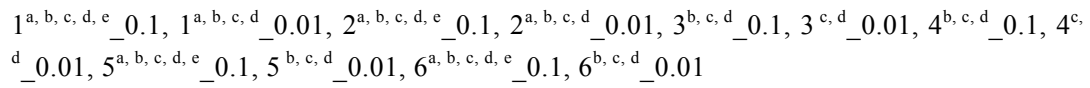 \\
\hline 38 & 33145927 & 9 & 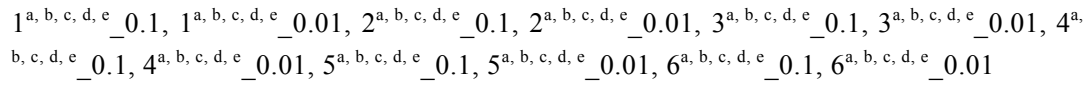 \\
\hline 39 & 33148740 & 9 & 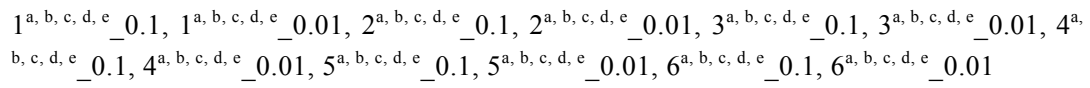 \\
\hline 40 & 33156262 & 9 & 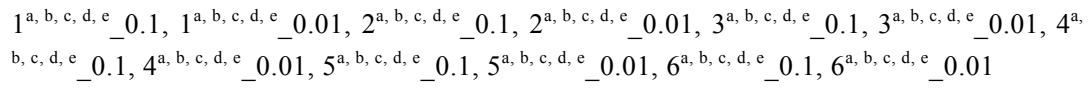 \\
\hline 41 & 33170290 & 9 & $1_{-}^{\mathrm{d}}-0.1,2^{\mathrm{d}}{ }_{-} 0.1,5^{\mathrm{d}}{ }_{-}^{0.1}, 6^{\mathrm{d}}-0.1$ \\
\hline 42 & 33170293 & 9 & $1_{-}^{\mathrm{d}} 0.1,2^{\mathrm{d}}{ }_{-}^{\mathrm{d}} 0.1,5^{\mathrm{d}}{ }_{-} 0.1,6^{\mathrm{d}}-0.1$ \\
\hline 43 & 33211574 & 9 & 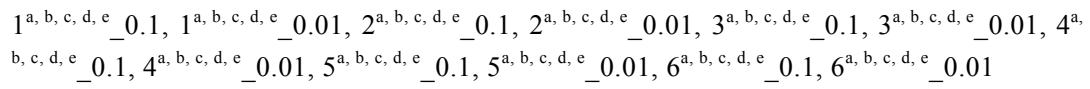 \\
\hline 44 & 33222811 & 9 & $\begin{array}{l}1^{\mathrm{c}, \mathrm{d}}-0.1,1^{\mathrm{d}}{ }_{-}^{\mathrm{d}} 0.01,2^{\mathrm{c}, \mathrm{d}}-0.1,2^{\mathrm{d}}-0.01,3^{\mathrm{d}}-0.1,3^{\mathrm{d}}-0.01,4^{\mathrm{d}}-0.1,4^{\mathrm{d}}-0.01,5^{\mathrm{c}, \mathrm{d}}-0.1,5^{\mathrm{d}}{ }_{-} 0.01, \\
6^{\mathrm{d}}-0.1,6^{\mathrm{d}}{ }^{0} 0.01\end{array}$ \\
\hline 45 & 33229209 & 9 & $1^{\mathrm{d}}-0.1,2^{\mathrm{d}}-_{0} 0.1,5^{\mathrm{d}}{ }_{-} 0.1$ \\
\hline 46 & 33230719 & 9 & $3^{\mathrm{d}}-_{0} 0.1,4^{\mathrm{d}}-0.1,5^{\mathrm{d}}-_{0.1}, 6^{\mathrm{d}}-0.1$ \\
\hline 47 & 33243331 & 9 & $\begin{array}{l}1^{\mathrm{d}}-0.1,1^{\mathrm{d}}{ }_{-}^{\mathrm{d}} 0.01,2^{\mathrm{d}}{ }_{-} 0.1,2^{\mathrm{d}}-0.01,3^{\mathrm{d}}-0.1,3^{\mathrm{d}}{ }_{-} 0.01,4^{\mathrm{d}}{ }_{-} 0.1,4^{\mathrm{d}}-0.01,5^{\mathrm{d}}-0.1,5^{\mathrm{d}}{ }_{-} 0.01,6^{\mathrm{d}}{ }_{-} 0.1, \\
6^{\mathrm{d}}-0.01\end{array}$ \\
\hline 48 & 33244698 & 9 & 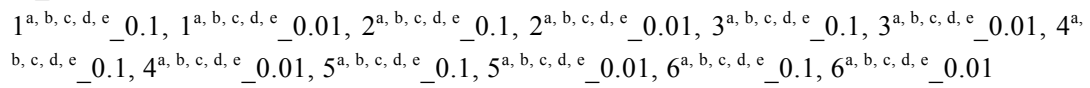 \\
\hline 49 & 33283731 & 9 & 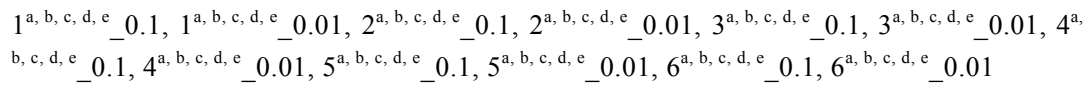 \\
\hline 50 & 33283949 & 9 & 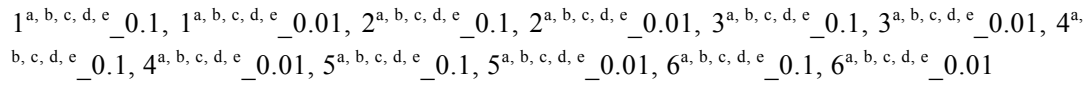 \\
\hline 51 & 33288990 & 9 & $1^{\mathrm{d}}-_{0.1}, 2^{\mathrm{d}}-0.1,3^{\mathrm{d}}-_{0} 0.1,4^{\mathrm{d}}{ }_{-} 0.1,5^{\mathrm{d}}-0.1,6^{\mathrm{d}}{ }_{-} 0.1$ \\
\hline 52 & 33302511 & 9 & $\begin{array}{l}1^{\mathrm{b}, \mathrm{c}, \mathrm{d}}-0.1,1^{\mathrm{d}}{ }^{\mathrm{d}} 0.01,2^{\mathrm{b}, \mathrm{c}, \mathrm{d}}-0.1,2^{\mathrm{d}}-0.01,3^{\mathrm{b}, \mathrm{c}, \mathrm{d}}-0.1,3^{\mathrm{d}}{ }_{-} 0.01,4^{\mathrm{b}, \mathrm{c}, \mathrm{d}}-0.1,4^{\mathrm{d}}-0.01,5^{\mathrm{b}, \mathrm{c}, \mathrm{d}}-0.1, \\
5^{\mathrm{d}}{ }_{-} 0.01,6^{\mathrm{b}, \mathrm{c}, \mathrm{d}}-0.1,6^{\mathrm{d}}{ }_{-} 0.01\end{array}$ \\
\hline 53 & 33451096 & 9 & 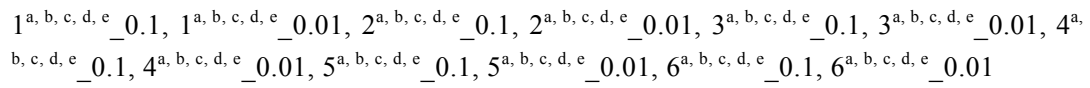 \\
\hline 54 & 33451119 & 9 & $\begin{array}{l}1^{\mathrm{b}, \mathrm{c}, \mathrm{d}}-0.1,1^{\mathrm{d}}{ }_{-} 0.01,2^{\mathrm{b}, \mathrm{c}, \mathrm{d}}-0.1,2^{\mathrm{d}}{ }_{-} 0.01,3^{\mathrm{d}} 0.1,3^{\mathrm{d}}{ }_{-} 0.01,4_{-}^{\mathrm{d}} 0.1,4_{-}^{\mathrm{d}} 0.01,5^{\mathrm{b}, \mathrm{c}, \mathrm{d}}-0.1, \\
5^{\mathrm{d}} 0.01,6^{\mathrm{b}, \mathrm{c}, \mathrm{d}}-0.1,6^{\mathrm{d}}{ }_{-} 0.01\end{array}$ \\
\hline 55 & 33480937 & 9 & 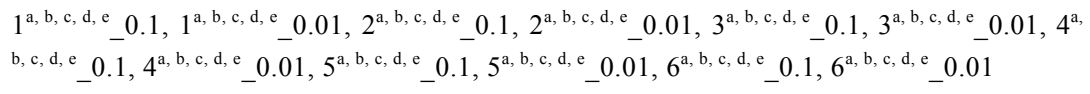 \\
\hline 56 & 34878453 & 9 & $3^{\mathrm{d}}-0.1,3^{\mathrm{d}}-_{0} 0.01,4_{-}^{\mathrm{d}} 0.1,4^{\mathrm{d}}-0.01,5^{\mathrm{d}}{ }_{-} 0.1,6^{\mathrm{d}}{ }_{-} 0.1,6^{\mathrm{d}}-0.01$ \\
\hline 57 & 34898663 & 9 & 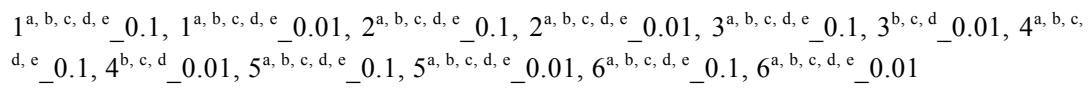 \\
\hline 58 & 34898695 & 9 & $\begin{array}{l}1^{\mathrm{a}, \mathrm{b}, \mathrm{c}, \mathrm{d}, \mathrm{e}} 0.1,1^{\mathrm{a}, \mathrm{b}, \mathrm{c}, \mathrm{d}, \mathrm{e}}-0.01,2^{\mathrm{a}, \mathrm{b}, \mathrm{c}, \mathrm{d}, \mathrm{e}}-0.1,2^{\mathrm{a}, \mathrm{b}, \mathrm{c}, \mathrm{d}, \mathrm{e}}-0.01,3^{\mathrm{b}, \mathrm{c}, \mathrm{d}, \mathrm{e}}-0.1,3^{\mathrm{b}, \mathrm{c}, \mathrm{d}} 0.01,4^{\mathrm{b}, \mathrm{c}, \mathrm{d},}-0 . \\
\mathrm{e}\end{array}$ \\
\hline 59 & 34908783 & 9 & 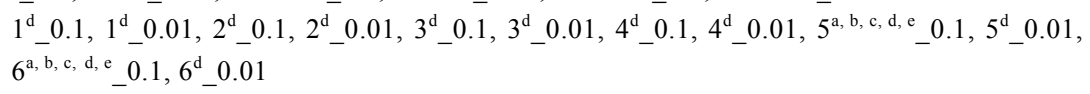 \\
\hline 60 & 34922341 & 9 & $1^{\mathrm{d}}-0.1,1^{\mathrm{d}}-0.01,2^{\mathrm{d}}-0.1,2^{\mathrm{d}}-0.01,3{ }^{\mathrm{d}}-0.1,4{ }^{\mathrm{d}}-0.1,5^{\mathrm{d}}-0.1,5^{\mathrm{d}}-0.01,6^{\mathrm{d}}-0.1,6^{\mathrm{d}}-0.01$ \\
\hline 61 & 34935499 & 9 & 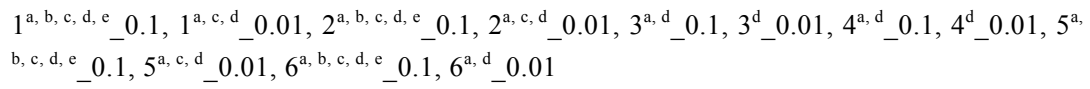 \\
\hline 62 & 34959324 & 9 & 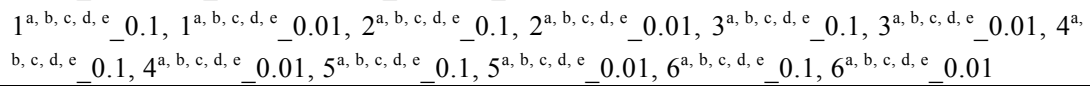 \\
\hline
\end{tabular}


(续表 2)

\begin{tabular}{|c|c|c|c|}
\hline $\begin{array}{c}\text { 编号 } \\
\text { Number }\end{array}$ & $\begin{array}{l}\text { SNP 位点 } \\
\text { SNP locus }\end{array}$ & $\begin{array}{l}\text { 染色体 } \\
\text { Chr. }\end{array}$ & $\begin{array}{c}\text { 不同模型、显著水平下与 SNP 相关联的性状 } \\
\text { Traits correlated with SNP under different analytical models and significant level }\end{array}$ \\
\hline 63 & 34964634 & 9 & $1_{-}^{\mathrm{d}}-0.1,2_{-}^{\mathrm{d}} 0.1$ \\
\hline 64 & 34998000 & 9 & $1^{\mathrm{d}}-0.1,2^{\mathrm{d}}{ }_{-}^{\mathrm{d}} 0.1,5^{\mathrm{d}}{ }_{-} 0.1,6^{\mathrm{d}}{ }_{-} 0.1$ \\
\hline 65 & 35003777 & 9 & 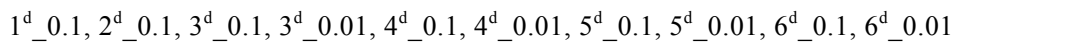 \\
\hline 66 & 35060347 & 9 & 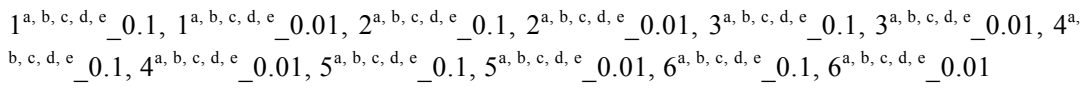 \\
\hline 67 & 35065836 & 9 & 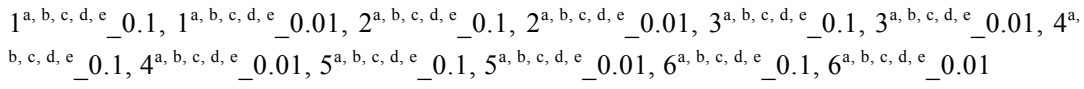 \\
\hline 68 & 35097925 & 9 & $\begin{array}{l}1_{-}^{\mathrm{d}} 0.1,1_{-}^{\mathrm{d}} 0.01,2_{-}^{\mathrm{d}} 0.1,2^{\mathrm{d}}-0.01,3_{-}^{\mathrm{d}} 0.1,3^{\mathrm{d}}{ }_{-} 0.01,4_{-}^{\mathrm{d}} 0.1,4_{-}^{\mathrm{d}}-0.01,5^{\mathrm{d}}-0.1,5^{\mathrm{d}}-0.01,6^{\mathrm{d}}{ }_{-} 0.1, \\
6^{\mathrm{d}} 0.01\end{array}$ \\
\hline 69 & 35097926 & 9 & $3^{\mathrm{d}}-0.1,4_{-}^{\mathrm{d}}-0.1,5^{\mathrm{d}}-0.1,6^{\mathrm{d}}-_{0.1}$ \\
\hline 70 & 35114336 & 9 & $3^{\mathrm{d}}-0.1,3^{\mathrm{d}}{ }_{-}^{\mathrm{d}} 0.01,4^{\mathrm{d}}{ }_{-} 0.1,4^{\mathrm{d}}-0.01,5^{\mathrm{d}}-0.1,6^{\mathrm{d}}{ }_{-} 0.1$ \\
\hline 71 & 35117629 & 9 & $\begin{array}{l}1^{\mathrm{a}, \mathrm{b}, \mathrm{c}, \mathrm{d}, \mathrm{e}}-0.1,1^{\mathrm{a}, \mathrm{c}, \mathrm{d}, \mathrm{e}}-0.01,2^{\mathrm{a}, \mathrm{b}, \mathrm{c}, \mathrm{d}, \mathrm{e}}-0.1,2^{\mathrm{a}, \mathrm{c}, \mathrm{d}, \mathrm{e}}-0.01,3^{\mathrm{a}, \mathrm{d}, \mathrm{e}}-0.1,3^{\mathrm{d}}-0.01,4^{\mathrm{a}, \mathrm{b}, \mathrm{d}, \mathrm{e}}-0.1,-{ }^{-}-1, \\
4^{\mathrm{d}}-0.01,5^{\mathrm{a}, \mathrm{b}, \mathrm{c}, \mathrm{d}, \mathrm{e}}-0.1,5^{\mathrm{a}, \mathrm{c}, \mathrm{d}, \mathrm{e}}-0.01,6^{\mathrm{a}, \mathrm{b}, \mathrm{c}, \mathrm{d}, \mathrm{e}}-0.1,6^{\mathrm{a}, \mathrm{d}}-0.01\end{array}$ \\
\hline 72 & 35123523 & 9 & 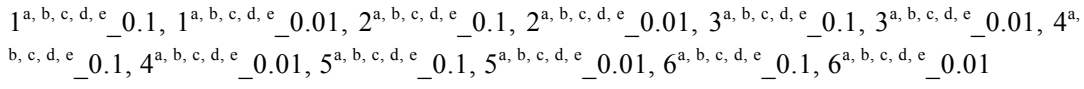 \\
\hline 73 & 35123542 & 9 & 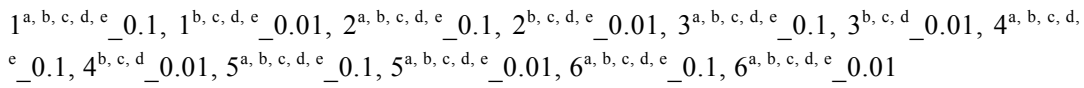 \\
\hline 74 & 35123599 & 9 & $3_{-}^{\mathrm{d}} 0.1,5_{-}^{\mathrm{d}} 0.1,6_{-}^{\mathrm{d}} 0.1$ \\
\hline 75 & 35180852 & 9 & $1^{\mathrm{d}}-0.1,2^{\mathrm{d}}{ }_{-}^{0.1}, 3^{\mathrm{d}}-0.1,4_{-}^{\mathrm{d}}-0.1,5^{\mathrm{d}}{ }_{-}^{0.1}, 6^{\mathrm{d}}-0.1$ \\
\hline 76 & 35212385 & 9 & 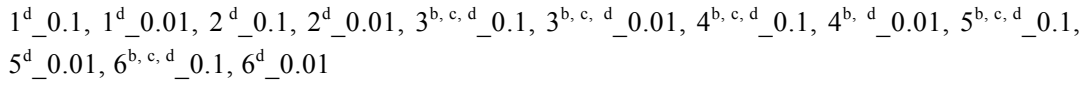 \\
\hline 77 & 35212534 & 9 & 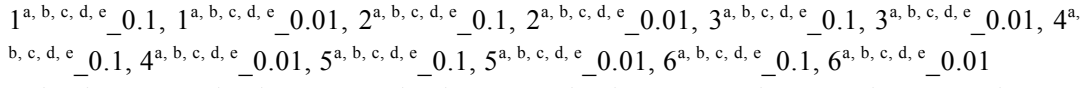 \\
\hline 78 & 35253233 & 9 & 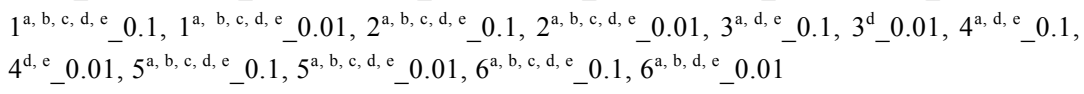 \\
\hline 79 & 35254558 & 9 & 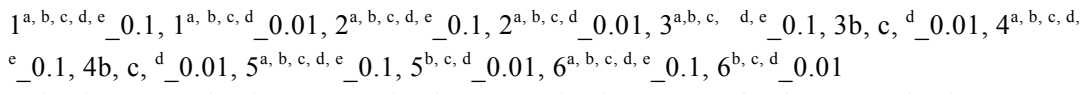 \\
\hline 80 & 35256410 & 9 & 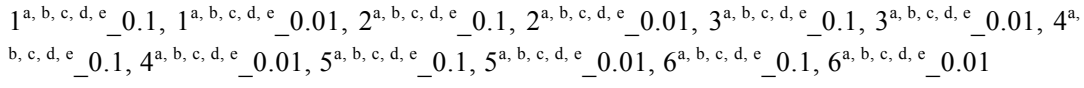 \\
\hline 81 & 8842711 & 11 & 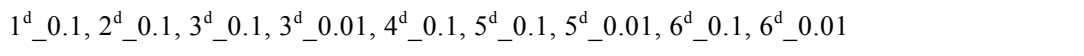 \\
\hline 82 & 23995788 & 12 & 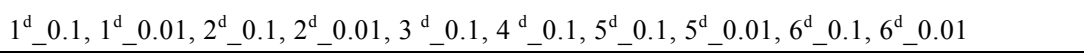 \\
\hline
\end{tabular}

1: AUDPC_2015; 2: SAUDPC_2015; 3: AUDPC_2016; 4: sAUDPC_2016; 5: AUDPC_mean; 6: sAUDPC_mean; a: CMLM 模型; b: EMMAX 模型; c: FASTLMM 模型; d: GLM 模型; e: MLM 模型; 显著水平分别为 0.1 和 0.01 。

1: AUDPC_2015; 2: sAUDPC_2015; 3: AUDPC_2016; 4: sAUDPC_2016; 5: AUDPC_mean; 6: sAUDPC_mean; a: CMLM; b: EMMAX; c: FASTLMM; d: GLM; e: MLM; significant difference at the 0.1 and 0.01 probability levels.

同源基因 $(R 8)$ 和 Rpi-vnt1 以及编码多效性耐药蛋白 基因; 5 个基因编码 MAPK 蛋白和 WRKY 转录因子; 1 个基因参与茉莉酸途径; 3 个基因与水杨酸途径相 关； 6 个基因是病程相关的基因； 3 个基因参与苯基 丙酸类合成途径; 其他与晚疫病抗性相关的基因如 HMGR 基因(2 个)、细胞色素 P450 (21 个)。

\section{3 讨论}

\section{1 不同模型分析对全基因关联分析结果的影} 响

本研究采用了 5 种模型对基因组数据和表型数 据进行关联分析。GLM 模型(general linear model)
采用 $\mathrm{Q}$ 矩阵 ${ }^{[16]}, \mathrm{MLM}$ 模型(mixed linear model)采用 $\mathrm{Q}+\mathrm{K}$ 矩阵 ${ }^{[16]}$, CMLM 模型 (compressed mixed linear model)算法介于 GLM 和 MLM 之间, 寻找最优算法 压缩计算时间 ${ }^{[20]}$, EMMAX 模型(efficient mixed model association eXpedited)在 MLM 模型基础上校 正关联群体的群体结构和遗传亲缘关系并加速这种 运算过程 ${ }^{[21-22]}$, FASTLMM (factored spectrally transformed linear mixed model)同样修正了 MLM 模型的 运算公式，提高运算速度 ${ }^{[23]}$ 。尽管不同的模型存在 差异, 但是在所有模型下分析的表型性状得到的结 果都比较可靠(图 2)。5 种模型下共关联到 82 个 SNP 位点, 而同一性状、同样的显著水平下, GLM 

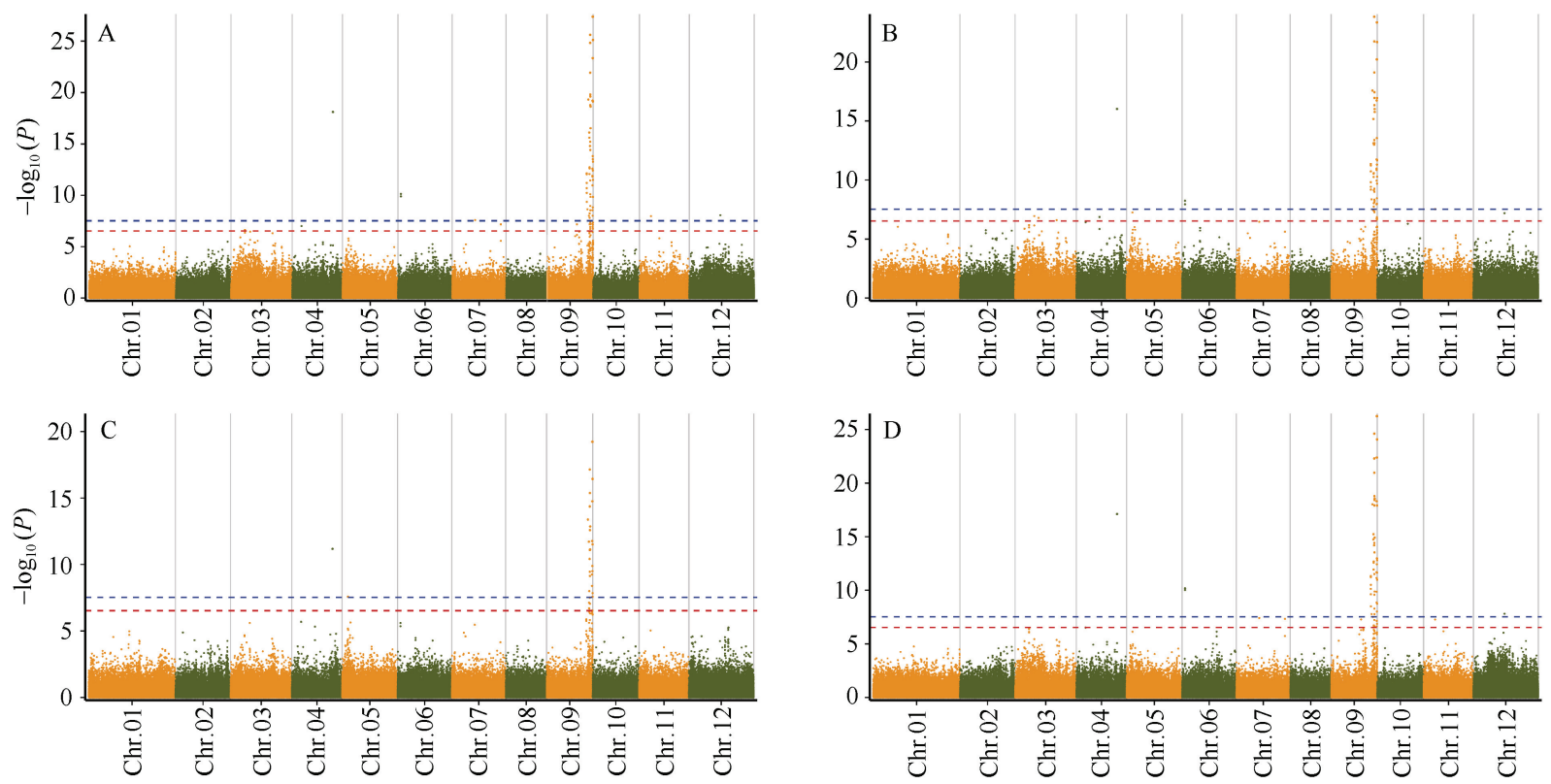

图 3 GWAS 分析生成的曼哈顿图

Fig. 3 Manhanttan plots of GWAS

A: GLM 模型分析 AUDPC_mean 性状; B: GLM 模型分析 AUDPC_16 性状; C: EMMAX 模型分析 sAUDPC_16 性状; D: GLM 模型分析 AUDPC_15 性状。

A: AUDPC_mean using GLM model; B: AUDPC_16 using GLM model; C: sAUDPC_16 using EMMAX; D: AUDPC_15 using GLM model.

模型分析关联到的 SNP 位点数量明显多于其他模型 (表 2)。与晚疫病抗性相关的候选基因中，关联到 48 个 SNP 位点, 其中 13 个(第 6、14、16、31、32、 $56 、 60 、 65 、 68 、 69 、 70 、 75$ 和 81 号) SNP 位点只 在 GLM 模型下关联到, 16 个 SNP 位点在所有模型 到关联到, 剩余的 19 个 SNP 位点在 2 4 个模型下获 得。因此, 利用多种模型分析能关联到更多可能与 晚疫病抗性相关的候选基因。

\section{2 马铃薯晚疫病水平抗性群体中可能存在的} 抗性基因

栽培马铃薯晚疫病抗性主要来自安第斯栽培种 S. tuberosum Andigenum group (包括 andigena、

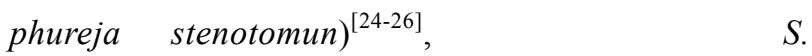
demissum $^{[27]} 、 S$. venturii $^{[7]} 、 S$. bulbocastanum ${ }^{[28]}{ }^{\text {等 }}$ 的抗性基因不断改良马铃薯栽培种的晚疫病抗性。 尽管国际马铃薯中心通过不断的轮回选择创制了一 批晚疫病水平抗性群体 ${ }^{[29]}$, 但是这批材料中可能仍 存在垂直抗性基因 $(R 1-R 11)^{[30]}$ 。本研究所用的试验 材料即是来自国际马铃薯中心篮选的水平抗性群体, 并且关联到 12 个 $R 1$ 类似基因, 3 个番茄斑萎病抗性 基因, 4 个 Rpi-vnt1 抗性基因以及 4 个多效性耐药蛋 白基因。

植物中抗性基因编码的最大的一类蛋白具有核 苷酸结合位点和亮氨酸重复结构(nucleotide-binding site plus leucine-rich repeat, NB-LRR) ${ }^{[31]}$, 而目前已 知的马铃薯晚疫病抗性基因编码的都是 NB-LRR 蛋 白 $^{[32]}$ 。 $R 1$ 基因定位在 5 号染色体上, 是植物抗性基 因亮氨酸 zipper/NBS/LRR 中的一员 ${ }^{[33]}$ 。根据前人的 研究, 至少有 4 个抗性基因位于 9 号染色体, 分别是 $R 8^{[8-9]} 、 R 9 a^{[34]}$ 、Rpi-vnt $1^{[7]}$ 和 $R p i-m o c I^{[35]}$, 这 4 个基 因均位于 9 号染色体长臂末端, 而且遗传距离比较 近 $^{[34,36]}$ 。本研究所关联到的 12 个 $R 1$ 类似基因位于 9 号染色体, 其长度为 $35.34 \mathrm{Mb}$, 而关联到的 SNP 的位置在 $34.8 \mathrm{Mb}$ 左右, 因此, 这些抗性基因肯定 不是 $R 1$, 而可能是已克隆的 $R 8$ 或其他抗性基因。 $S w-5$ 是番茄中一类广谱抗性的番茄斑萎病( ToMV) 抗性基因 ${ }^{[37]}$, 位于 9 号染色体上的抗性基因 $R 8$ 与 $S w-5$ 同源, 而 Rpi-vnt1 与番茄中另 1 个 ToMV 持久 抗性基因 $\mathrm{Tm}-2^{2}$ 同源 ${ }^{[7,38]}$ 。本研究中另外关联到与 $R P i$-vnt 1 基因类似的基因, 其位置在 $32.5 \mathrm{Mb}$ 附近, 而关联到的 ToMV 抗性基因位于 $34.7 \mathrm{Mb}$ 左右, 根 据 $R 8$ 与 $R p i-v n t 1$ 的遗传位置 ${ }^{[36]}, R p i-v n t 1$ 在 $R 8$ 的上 方, 因此关联到位于 $32.5 \mathrm{Mb}$ 的基因可能是 Rpi-vnt 1 , 而位于 $34.7 \mathrm{Mb}$ 的基因可能是 $R 8$ 。多效性耐药蛋白 是一类 $\mathrm{ABC}$ 转运蛋白, 可参与到拟南芥 ${ }^{[39]}(P E N 3)$ 和小麦 ${ }^{[40]}(L r 34)$ 中非宿主抗性和对病原菌的持久抗 


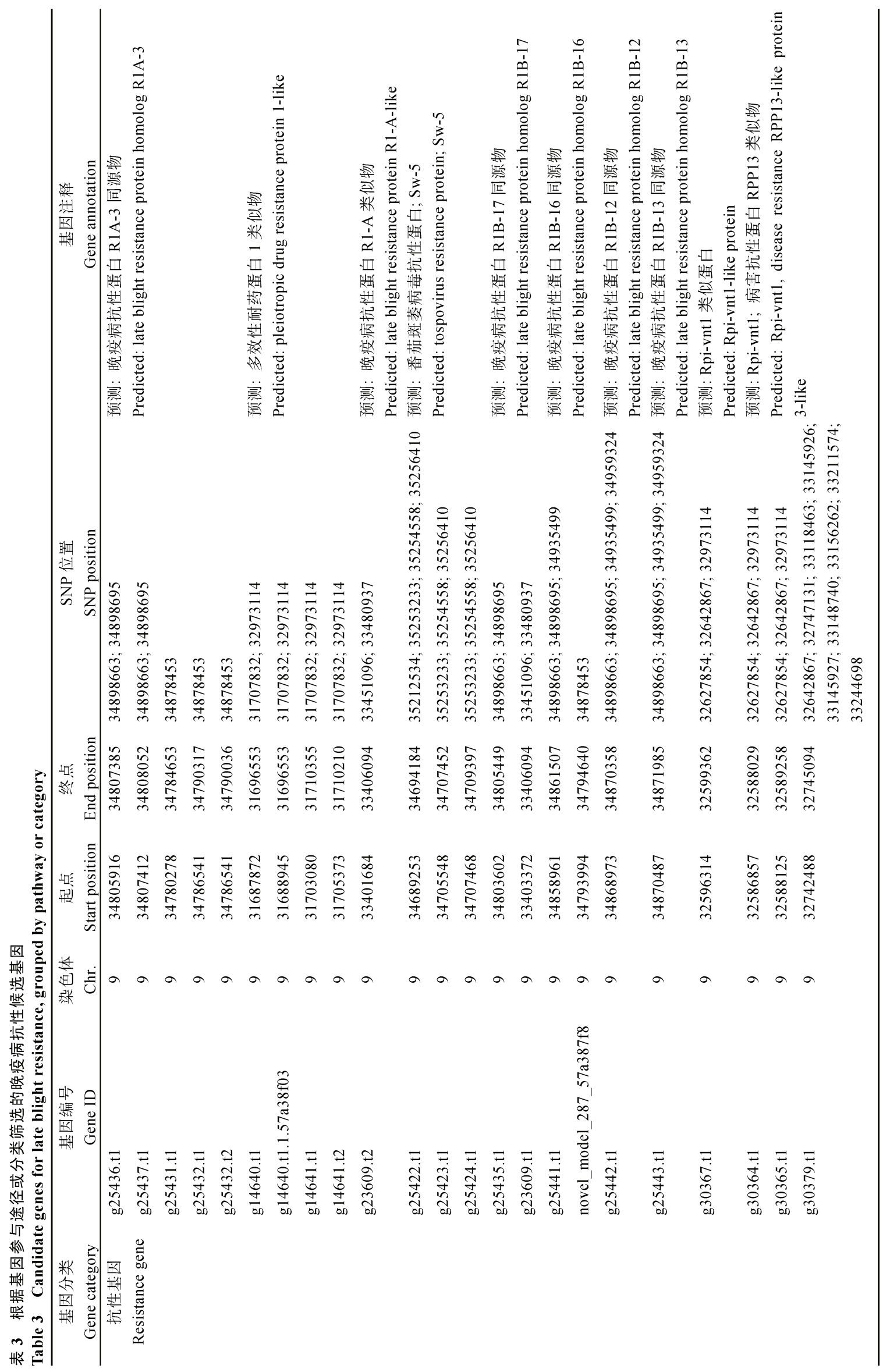




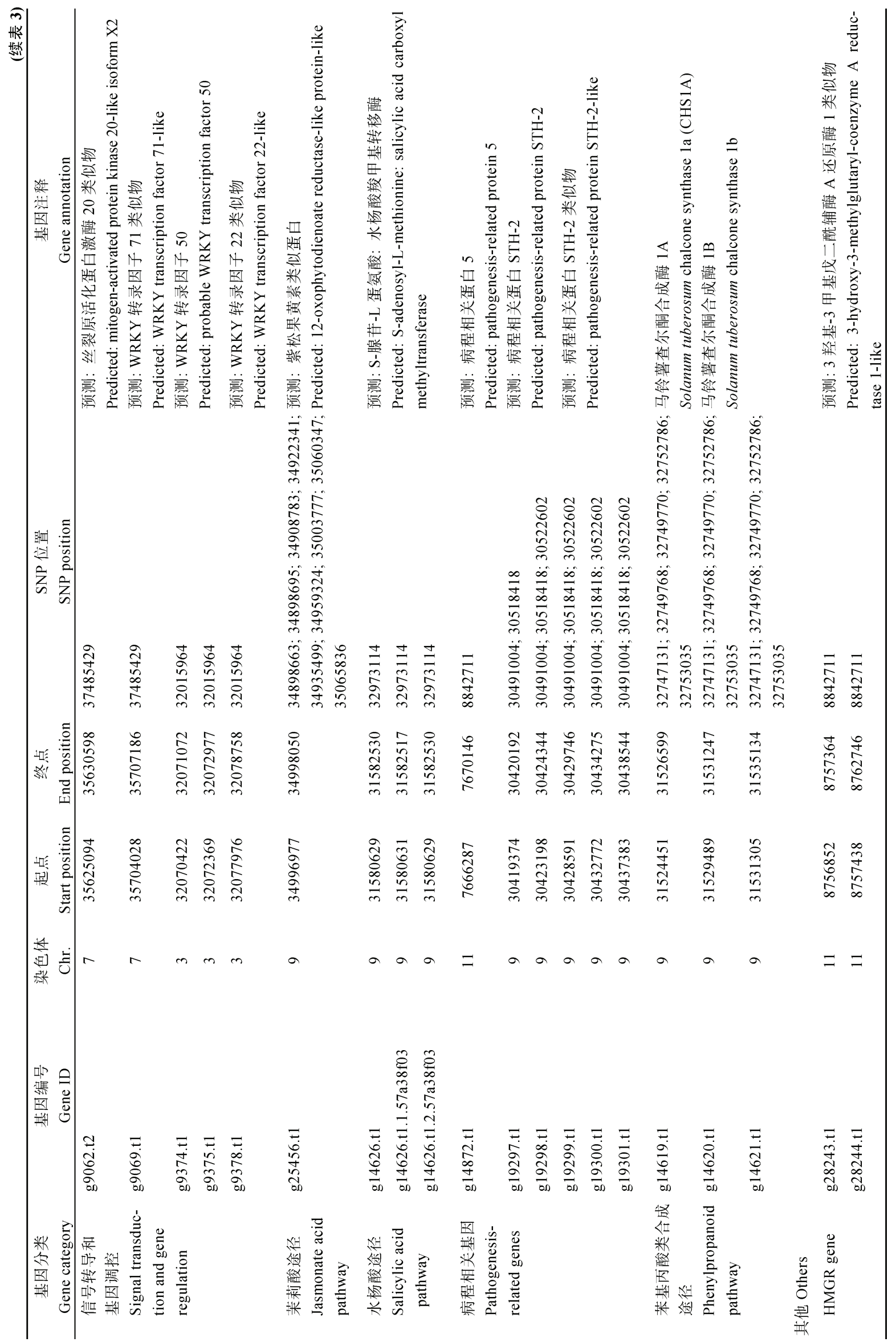


刑
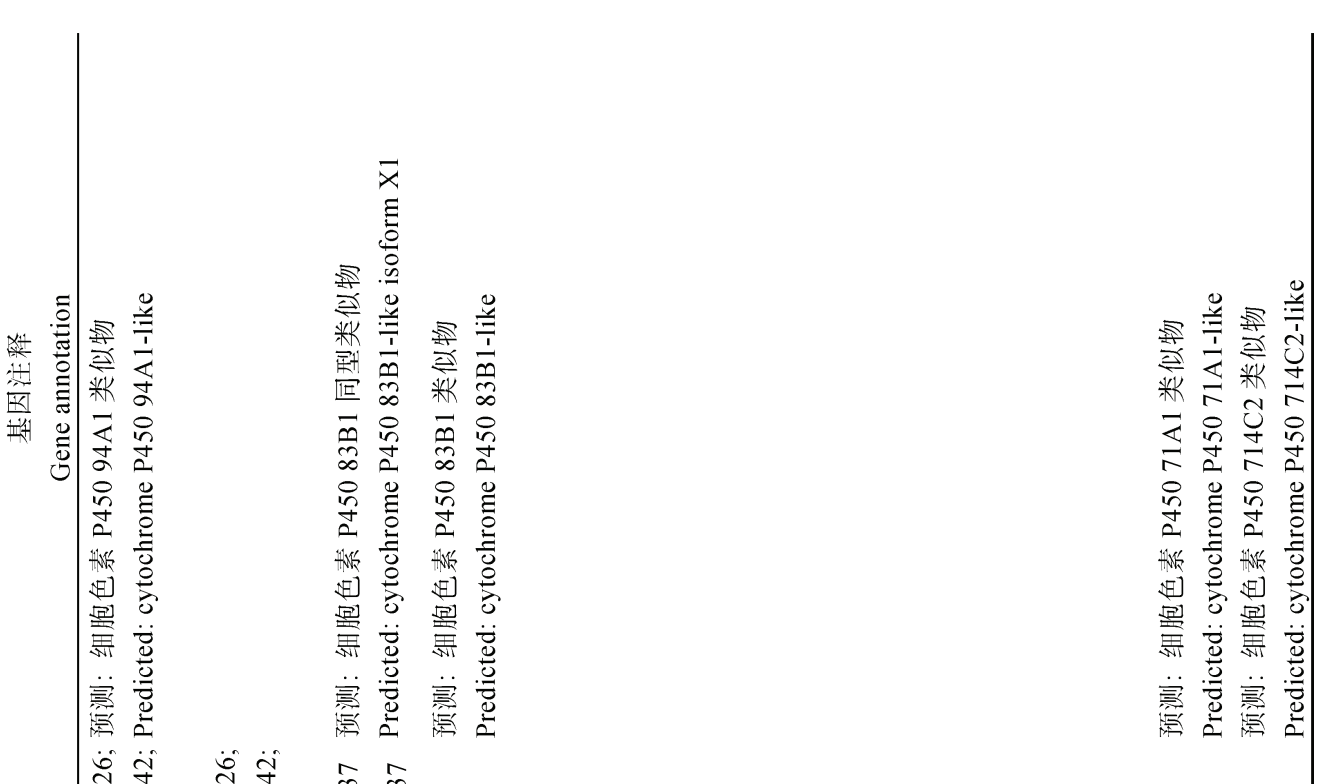

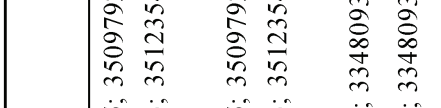

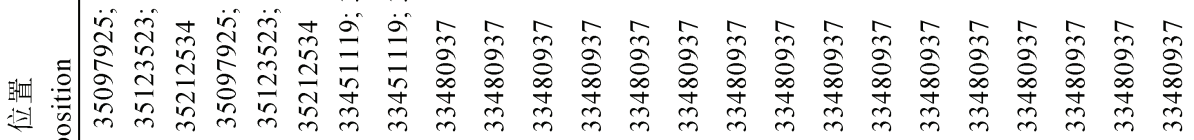

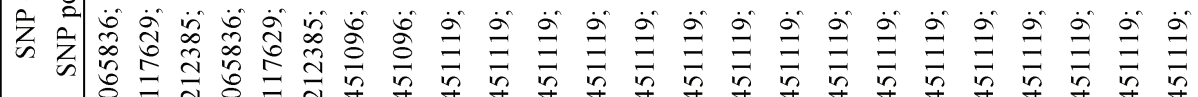

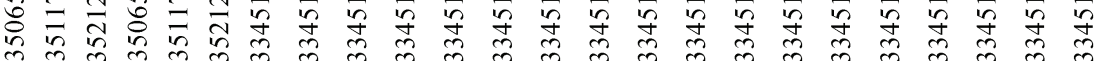

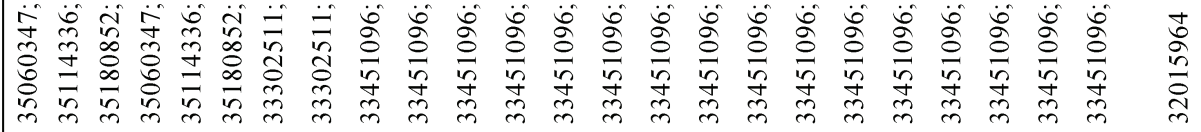

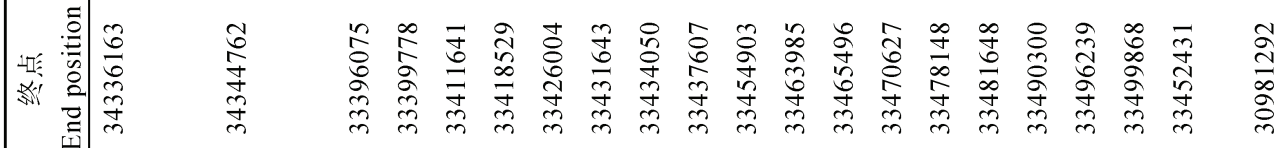

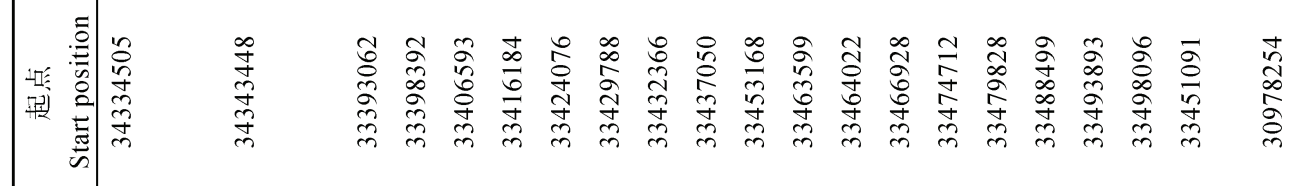

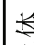

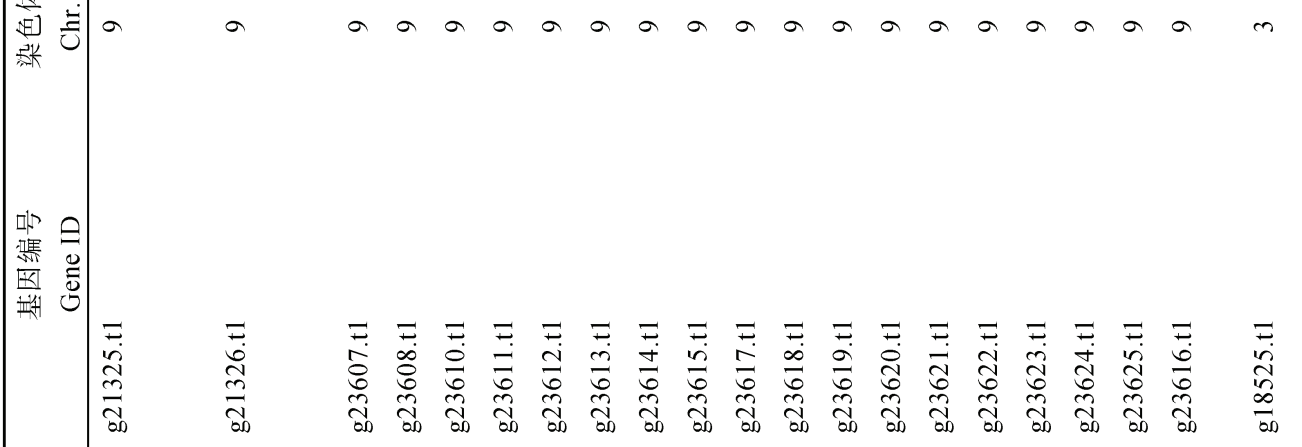

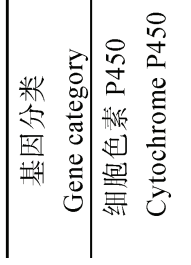


性中。马铃薯中的多效性耐药蛋白也可参与到晚疫 病菌的侵染过程中, 但是具体怎么调节这个过程尚 不清楚 ${ }^{[41]}$ 。本研究关联到的多效性耐药蛋白基因位 于 $31.7 \mathrm{Mb}$ 左右, 其对马铃薯晚疫病抗性贡献的大 小, 有待进一步研究。

\section{3 其他可能影响马铃薯晚疫病抗性的相关基}

因

本研究所用材料主要是表现出晚疫病水平抗性, 即非 $R$ 基因主导的持久抗性 ${ }^{[29]}$ 。还存在其他途径影 响晚疫病抗性 ${ }^{[42-43]}$, 包括参与信号转导和基因调 控、茉莉酸途径、水杨酸途径、病程相关基因、苯 基丙酸类合成途径、HMGR 基因和细胞色素 P450。 马铃薯中 MAPK 激酶和 WRKY 转录因子可参与植物

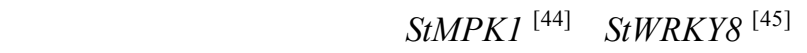
StWRKY1 ${ }^{[46]}$ 。本研究在 7 号染色体上关联到 1 个 $M A P K$ 基因和 $W R K Y$ 基因, 在 3 号染色体上关联到 3 个 $W R K Y$ 基因, 它们对晚疫病水平抗性的贡献有待 进一步研究。激素信号在植物病害防御中发挥着重 要作用, 植物激素茉莉酸和水杨酸代谢途径中的因 子不同程度地参与到马铃薯晚疫病水平抗性中 ${ }^{[47-49]}$ 。 本研究中关联到 1 个与茉莉酸代谢途径可能相关和 3 个与水杨酸代谢途径可能相关的基因, 在其他马 铃薯晚疫病抗性全基因组关联分析和 QTL 定位研 究中也定位到与茉莉酸和水杨酸代谢途径相关的 基因 ${ }^{[50-51]}$ 。有趣的是, 本研究中关联到 3 个参与苯 基丙酸类合成途径的查尔酮合成酶基因 $(C H S)$, 位 于 9 号染色体。马铃薯中 $C H S$ 基因至少有 6 个拷贝, 研究较多的是位于 5 号染色体的 $C H S$ 基因参与马铃 薯块茎中花青素合成 ${ }^{[52-54]}$ 。但是, 查尔酮合成酶能 够参与植物病害防御反应 ${ }^{[55-56]}$, 表明位于 9 号染色 体的 CHS 基因可能是构成马铃薯晚疫病水平抗性因 素之一。其他基因包括与病程相关的基因、HMGR 基因和细胞色素 P450 都有报道可以参与植物病害 防御过程, 但是在马铃薯晚疫病水平抗性中所起的 作用是不清楚的, 而本研究关联到的基因可以为马 铃薯晚疫病水平抗性的形成机理提供参考 ${ }^{[42,57-59]}$ 。

\section{4 结论}

基于 5 种模型的 GWAS 结果，共检测到 82 个与 晚疫病抗性显著相关的 SNP 位点, 其中 48 个位点关 联到可能与晚疫病抗性相关的候选基因，包括 $R 1$ 同 源基因、 $S w-5$ 同源基因 $(R 8) 、 R p i-v n t 1$ 基因、与信 号转导相关的 $M A P K$ 基因、WRKY 基因、参与植物 激素茉莉酸和水杨酸代谢相关的基因、参与苯基丙
酸类合成途径的查尔酮酶基因和其他病程相关的基 因、HMGR 基因以及细胞色素 $P 450$ 基因。

\section{References}

[1] 吴秋云, 黄科, 刘明月, 周倩, 熊兴耀. 马铃薯晚疫病抗病基 因研究进展. 中国马铃薯, 2014, 28: 175-179.

Wu Q Y, Huang K, Liu M Y, Zhou Q, Xiong X Y. Research progress of late blight resistance genes in potato. China Potato J, 2014, 28: 175-179 (in Chines with English abstract).

[2] 李亚红, 赵俊, 金吉斌, 石安宪, 马永翠, 桂富荣. 云南省马 铃薯晚疫病发生原因分析及治理对策. 中国植保导刊, 2014, 34(9): 22-24.

Li Y H, Zhao J, Jin J B, Shi A X, Ma Y C, Gui F R. Cause analysis and control countermeasure of potato late blight in Yunnan province. China Plant Prot, 2014, 34(9): 22-24 (in Chinese).

[3] Martin M D, Cappellini E, Samaniego J A, Zepeda M L, Campos P F, Seguin-Orlando A, Wales N, Orlando L, Ho S Y W, Dietrich F S, Mieczkowski P A, Heitman J, Willerslev E, Krogh A, Ristaino J B, Gilbert M T P. Reconstructing genome evolution in historic samples of the Irish potato famine pathogen. Nat Commun, 2013, 4: 2172-2178.

[4] van der Vossen E A G, Gros J, Sikkema A, Muskens M, Wouters D, Wolters P, Pereira A, Allefs S. The Rpi-blb2 gene from Solanum bulbocastanum is a Mi-1 gene homolog conferring broad-spectrum late blight resistance in potato. Plant J, 2005, 44: 208-222.

[5] Song J, Bradeen J M, Naess S K, Raasch J A, Wielgus S M, Haberlach G T, Liu J, Kuang H, Austin-Phillips S, Buell C R, Helgeson J P, Jiang J. Gene RB cloned from Solanum bulbocastanum confers broad spectrum resistance to potato late blight. Proc Natl Acad Sci USA, 2003, 100: 9128-9133.

[6] van der Vossen E, Sikkema A, Hekkert B T L, Gros J, Stevens P, Muskens M, Wouters D, Pereira A, Stiekema W, Allefs S. An ancient $R$ gene from the wild potato species Solanum bulbocastanum confers broad-spectrum resistance to Phytophthora infestans in cultivated potato and tomato. Plant J, 2003, 36: 867-882.

[7] Foster S J, Park T H, Pel M, Brigneti G, Śliwka J, Jagger L, van der Vossen E, Jones J D G. Rpi-vnt1.1, a Tm-2 $2^{2}$ homolog from Solanum venturii, confers resistance to potato late blight. $\mathrm{Mol}$ Plant-Microbe Interact, 2009, 22: 589-600.

[8] Jiang R, Li J, Tian Z D, Du J, Armstrong M, Baker K, Tze-Yin L J, Vossen J H, He H, Portal L, Zhou J, Bonierbale M, Hein I, Lindqvist-Kreuze $\mathrm{H}$, Xie $\mathrm{C} \mathrm{H}$. Potato late blight field resistance from QTL dPI09c is conferred by the NB-LRR gene R8. $J$ Exp Bot, 2018, 69: 1545-1555.

[9] Vossen J H, van Arkel G, Bergervoet M, Jo K R, Jacobsen E, Visser R G F. The Solanum demissum R8 late blight resistance gene is an $S w-5$ homologue that has been deployed worldwide in late blight resistant varieties. Theor Appl Genet, 2016, 129: 1785-1796.

[10] Lindqvist-Kreuze H, de Boeck B, Unger P, Gemenet D, Li X P, Pan Z C, Sui Q J, Qin J H, Woldegjorgis G, Negash K, Seid I, Hirut B, Gastelo M, de Vega J, Bonierbale M. Global multi-environment resistance QTL for foliar late blight resistance in tetraploid potato with tropical adaptation. BioRxiv, 2020. 
https://doi.org/10.1101/2020.02.16.950618.

[11] Leisner C P, Hamilton J P, Crisovan E, Manrique-Carpintero N C, Marand A P, Newton L, Pham G M, Jiang J, Douches D S, Jansky S H, Buell C R. Genome sequence of M6, a diploid inbred clone of the high-glycoalkaloid-producing tuber-bearing potato species Solanum chacoense, reveals residual heterozygosity. Plant $J$, 2018, 94: 562-570.

[12] Sun X, Liu D, Zhang X, Li W, Liu H, Hong W, Jiang C, Guan N, Ma C, Zeng H, Xu C, Song J, Huang L, Wang C, Shi J, Wang R, Zheng X, Lu C, Wang X, Zheng H. SLAF-seq: an efficient method of large-scale de novo SNP discovery and genotyping using high-throughput sequencing. PLoS One, 2013, 8: e58700.

[13] Li H, Durbin R. Fast and accurate short read alignment with Burrows-Wheeler transform. Bioinformatics, 2009, 25: 1754 1760.

[14] McKenna A, Hanna M, Banks E, Sivachenko A, Cibulskis K, Kernytsky A, Garimella K, Altshuler D, Gabriel S, Daly M. The genome analysis toolkit: a MapReduce framework for analyzing next-generation DNA sequencing data. Genome Res, 2010, 20: 1297-1303.

[15] Li H, Handsaker B, Wysoker A, Fennell T, Ruan J, Homer N, Marth G, Abecasis G, Durbin R. The sequence alignment/map format and SAMtools. Bioinformatics, 2009, 25: 2078-2079.

[16] Bradbury P J, Zhang Z, Kroon D E, Casstevens T M, Ramdoss Y, Buckler E S. TASSEL: software for association mapping of complex traits in diverse samples. Bioinformatics, 2007, 23: 2633-2635.

[17] Alexander D H, Novembre J, Lange K. Fast model-based estimation of ancestry in unrelated individuals. Genome Res, 2009, 19: 1655-1664.

[18] Hardy O J, Vekemans X. SPAGeDi: a versatile computer program to analyse spatial genetic structure at the individual or population levels. Mol Ecol Notes, 2002, 2: 618-620.

[19] Wickham H. ggplot2: Elegant Graphics for Data Analysis. New York: Springer Press, 2009. p 224.

[20] Zhang Z, Ersoz E, Lai C Q, Todhunter R J, Tiwari H K, Gore M A, Bradbury P J, Yu J, Arnett D K, Ordovas J M, Buckler E S. Mixed linear model approach adapted for genome-wide association studies. Nat Genet, 2010, 42: 355-360.

[21] Kang H M, Zaitlen N A, Wade C M, Kirby A, Heckerman D, Daly M J, Eskin E. Efficient control of population structure in model organism association mapping. Genetics, 2008, 178: 1709-1723.

[22] Kang H M, Sul J H, Service S K, Zaitlen N A, Kong S Y, Freimer N B, Sabatti C, Eskin E. Variance component model to account for sample structure in genome-wide association studies. Nat Genet, 2010, 42: 348-354.

[23] Lippert C, Listgarten J, Liu Y, Kadie C M, Davidson R I, Heckerman D. FaST linear mixed models for genome-wide association studies. Nat Methods, 2011, 8: 833-835.

[24] Spooner D M, Ghislain M, Simon R, Jansky S H, Gavrilenko T. Systematics, diversity, genetics, and evolution of wild and cultivated potatoes. Bot Rev, 2014, 80: 283-383.

[25] Ghislain M, Trognitz B, Del R. Herrera M, Solis J, Casallo G, Vásquez C, Hurtado O, Castillo R, Portal L, Orrillo M. Genetic loci associated with field resistance to late blight in offspring of
Solanum phureja and S. tuberosum grown under short-day conditions. Theor Appl Genet, 2001, 103: 433-442.

[26] Simko I, Costanzo S, Ramanjulu V, Christ B J, Haynes K G. Mapping polygenes for tuber resistance to late blight in a diploid Solanum phureja $\times$ S. stenotomum hybrid population. Plant Breed, 2006, 125: 385-389.

[27] Colon L T, Turkensteen L J, Prummel W, Budding D J, Hoogendoorn J. Durable resistance to late blight (Phytophthora infestans) in old potato cultivars. Eur J Plant Pathol, 1995, 101: 387-397.

[28] Naess S K, Bradeen J M, Wielgus S M, Haberlach G T, Mcgrath J M, Helgeson J P. Resistance to late blight in Solanum bulbocastanum is mapped to chromosome 8. Theor Appl Genet, 2000, 101: 697-704.

[29] Landeo J A, Gastelo M, Beltran G, Diaz L. Quantifying genetic variance for horizontal resistance to late blight in potato breeding population B3C1. In: The International Potato Center Program Report 1999-2000, Scientist and Farmer-Partners in Research for the 21st Century. Lima, Peru, 2001. pp 63-68.

[30] Lindqvist-Kreuze H, Gastelo M, Perez W, Forbes G A, de Koeyer $\mathrm{D}$, Bonierbale M. Phenotypic stability and genome-wide association study of late blight resistance in potato genotypes adapted to the tropical highlands. Phytopathology, 2014, 104: 624-633.

[31] Dangl J L, Jones J D G. Plant pathogens and integrated defence responses to infection. Nature, 2001, 411: 826-833.

[32] Marla S S. Structural analysis of resistance (R) genes in potato (Solanum Species) genome. In: Kumar Chakrabarti S, Xie C, Kumar Tiwari J, eds. The Potato Genome. Cham: Springer International Publishers, 2017. pp 269-281.

[33] Ballvora A, Ercolano M R, Weiß J, Meksem K, Bormann C A, Oberhagemann P, Salamini F, Gebhardt C. The Rl gene for potato resistance to late blight (Phytophthora infestans) belongs to the leucine zipper/NBS/LRR class of plant resistance genes. Plant $J$, 2002, 30: 361-371.

[34] Jo K-R, Visser R G F, Jacobsen E, Vossen J H. Characterisation of the late blight resistance in potato differential MaR9 reveals a qualitative resistance gene, $R 9 a$, residing in a cluster of Tm-2(2) homologs on chromosome IX. Theor Appl Genet, 2015, 128: 931-941.

[35] Smilde W D, Brigneti G, Jagger L, Perkins S, Jones J D G. Solanum mochiquense chromosome IX carries a novel late blight resistance gene Rpi-moc1. Theor Appl Genet, 2005, 110: $252-258$.

[36] Jo K R, Arens M, Kim T Y, Jongsma M A, Visser R G F, Jacobsen $\mathrm{E}$, Vossen J H. Mapping of the $S$. demissum late blight resistance gene $R 8$ to a new locus on chromosome IX. Theor Appl Genet, 2011, 123: 1331-1340.

[37] Brommonschenkel S H, Frary A, Frary A, Tanksley S D. The broad-spectrum tospovirus resistance gene $S w-5$ of tomato is a homolog of the root-knot nematode resistance gene $\mathrm{Mi}$. Mol Plant-Microbe Interact, 2000, 13: 1130-1138.

[38] Lanfermeijer F C, Dijkhuis J, Sturre M J G, de Haan P, Hille J. Cloning and characterization of the durable tomato mosaic virus resistance gene $T m-2^{2}$ from Lycopersicon esculentum. Plant Mol Biol, 2003, 52: 1039-1051.

[39] Stein M, Dittgen J, Sánchez-Rodríguez C, Hou B H, Molina A, Schulze-Lefert P, Lipka V, Somerville S. Arabidopsis 
PEN3/PDR8, an ATP binding cassette transporter, contributes to nonhost resistance to inappropriate pathogens that enter by direct penetration. Plant Cell, 2006, 18: 731-746.

[40] Krattinger S G, Lagudah E S, Spielmeyer W, Singh R P, Huerta-Espino J, Mcfadden H, Bossolini E, Selter L L, Keller B. A putative $\mathrm{ABC}$ transporter confers durable resistance to multiple fungal pathogens in wheat. Science, 2009, 323: 1360-1363.

[41] Ruocco M, Ambrosino P, Lanzuise S, Woo S L, Lorito M, Scala F. Four potato (Solanum tuberosum) ABCG transporters and their expression in response to abiotic factors and Phytophthora infestans infection. J Plant Physiol, 2011, 168: 2225-2233.

[42] Gebhardt C, Valkonen J P T. Organazation of gene controlling disease resistance in the potato genome. Annu Rev Phytopathol, 2001, 39: 79-102.

[43] Trognitz F, Manosalva P, Gysin R, Niño-Liu D, Simon R, del Rosario Herrera M, Trognitz B, Ghislain M, Nelson R. Plant defense genes associated with quantitative resistance to potato late blight in Solanum phureja $\times$ dihaploid S. tuberosum hybrids. Mol Plant-Microbe Interact, 2002, 15: 587-597.

[44] Yamamizo C, Kuchimura K, Kobayashi A, Katou S, Kawakita K, Jones J D G, Doke N, Yoshioka H. Rewiring mitogen-activated protein kinase cascade by positive feedback confers potato blight resistance. Plant Physiol, 2006, 140: 681-692.

[45] Yogendra K N, Dhokane D, Kushalappa A C, Sarmiento F, Rodriguez E, Mosquera T. StWRKY8 transcription factor regulates benzylisoquinoline alkaloid pathway in potato conferring resistance to late blight. Plant Sci, 2017, 256: 208-216.

[46] Yogendra K N, Kumar A, Sarkar K, Li Y, Pushpa D, Mosa K A, Duggavathi R, Kushalappa A C. Transcription factor StWRKY1 regulates phenylpropanoid metabolites conferring late blight resistance in potato. $J$ Exp Bot, 2015, 66: 7377-7389.

[47] Derksen H, Rampitsch C, Daayf F. Signaling cross-talk in plant disease resistance. Plant Sci, 2013, 207: 79-87.

[48] Saubeau G, Perrin F, Marnet N, Andrivon D, Val F. Hormone signalling pathways are differentially involved in quantitative resistance of potato to Phytophthora infestans. Plant Pathol, 2016, 65: 342-352.

[49] Halim V A, Eschen-Lippold L, Altmann S, Birschwilks M, Scheel D, Rosahl S. Salicylic acid is important for basal defense of Solanum tuberosum against Phytophthora infestans. Mol Plant-Microbe Interact, 2007, 20: 1346-1352.

[50] Mosquera T, Alvarez M F, Jiménez-Gómez J M, Muktar M S, Paulo M J, Steinemann S, Li J, Draffehn A, Hofmann A, Lübeck J, Strahwald J, Tacke E, Hofferbert H R, Walkemeier B, Gebhardt C. Targeted and untargeted approaches unravel novel candidate genes and diagnostic SNPs for quantitative resistance of the potato (Solanum tuberosum L.) to Phytophthora infestans causing the late blight disease. PLoS One, 2016, 11: e0156254.

[51] Danan S, Chauvin J E, Caromel B, Moal J D, Pelle R, Lefebvre V. Major-effect QTLs for stem and foliage resistance to late blight in the wild potato relatives Solanum sparsipilum and S. spegazzinii are mapped to chromosome X. Theor Appl Genet, 2009, 119: 705-719.

[52] Jeon J H, Kim H S, Choi K H, Joung Y H, Joung H, Byun S M. Cloning and characterization of one member of the chalcone synthase gene family from Solanum tuberosum L. Biosci Biotechnol Biochem, 1996, 60: 1907-1910.

[53] de Jong W S, Eannetta N T, de Jong D M, Bodis M. Candidate gene analysis of anthocyanin pigmentation loci in the Solanaceae. Theor Appl Genet, 2004, 108: 423-432.

[54] Zhang Y F, Jung C S, de Jong W S. Genetic analysis of pigmented tuber flesh in potato. Theor Appl Genet, 2009, 119: $143-150$.

[55] Dixon R A, Paiva N L. Stress-induced phenylpropanoid metabolism. Plant Cell, 1995, 7: 1085-1097.

[56] Dixon R A, Achnine L, Kota p, Liu C J, Reddy M S S, Wang L. The phenylpropanoid pathway and plant defence-a genomics perspective. Mol Plant Pathol, 2002, 3: 371-390.

[57] Kuc J. Phytoalexins, stress metabolism, and disease resistance in plants. Annu Rev Phytopathol, 1995, 33: 275-297.

[58] Du J, Tian Z D, Liu J, Vleeshouwers V, Shi X, Xie C H. Functional analysis of potato genes involved in quantitative resistance to Phytophthora infestans. Mol Biol Rep, 2013, 40: 957-967.

[59] Álvarez M F, Angarita M, Delgado M C, García C, Jiménez-Gomez J, Gebhardt C, Mosquera T. Identification of novel associations of candidate genes with resistance to late blight in Solanum tuberosum Group Phureja. Front Plant Sci, 2017, 8: 1-11. 